accepted for publication in ApJ

\title{
Dust Destruction in the High-Velocity Shocks Driven by Supernovae in the Early Universe
}

\author{
Takaya Nozawa and Takashi Kozasa \\ Division of Earth and Planetary Sciences, Graduate School of Science, Hokkaido \\ University, Sapporo 060-0810, Japan; nozawa@ep.sci.hokudai.ac.jp, \\ kozasa@ep.sci.hokudai.ac.jp \\ and \\ Asao Habe \\ Division of Physics, Graduate School of Science, Hokkaido University, Sapporo 060-0810, \\ Japan; habe@astro1.sci.hokudai.ac.jp
}

\begin{abstract}
We investigate the destruction of dust grains by sputtering in the high-velocity interstellar shocks driven by supernovae (SNe) in the early universe to reveal the dependence of the time-scale of dust destruction on the gas density $n_{\mathrm{H}, 0}$ in the interstellar medium (ISM) as well as on the progenitor mass $M_{\mathrm{pr}}$ and explosion energy $E_{51}$ of SN. The sputtering yields for the combinations of dust and ion species of interest to us are evaluated by applying the so-called universal relation with a slight modification. The dynamics of dust grains and their destruction by sputtering in shock are calculated by taking into account the size distribution of each dust species, together with the time evolution of temperature and density of gas in spherically symmetric shocks. The results of calculations show that the efficiency of dust destruction depends not only on the sputtering yield but also on the initial size distribution of each grain species. The efficiency of dust destruction increases with increasing $E_{51}$ and/or increasing $n_{\mathrm{H}, 0}$, but is almost independent of $M_{\mathrm{pr}}$ as long as $E_{51}$ is the same. The mass of gas swept up by shock is the increasing function of $E_{51}$ and the decreasing function of $n_{\mathrm{H}, 0}$. Combining these results, we present the approximation formula for the time-scale of destruction for each grain species in the early universe as a function of $E_{51}$ and $n_{\mathrm{H}, 0}$. This formula is applicable for investigating the evolution of dust grains at the early epoch of the universe with the metallicity of $Z \lesssim 10^{-3} Z_{\odot}$. The effects of the cooling processes of gas on the destruction of dust are briefly discussed.
\end{abstract}


Subject headings: dust: extinction, destruction - early universe- supernovae: population III - supernova remnant: plasma - interstellar medium: shock wave

\section{Introduction}

Population III (Pop III) stars formed from metal-free gas clouds where $\mathrm{H}_{2}$ molecule is the main coolant of gas are considered to be more massive than $10^{2} M_{\odot}$ (Bromm \& Larson 2004 and references therein), though there are still numerous uncertainties regarding the mass range of the first stars due to a poor understanding of the relevant radiative feedback in the late accretion phase (see for example Tan \& McKee 2004). Once the primordial gas is enriched by dust grains, dust have great influences on the subsequent formation and evolution history of stars and galaxies; the star formation rate (SFR) in the metal-poor star-forming clouds is enhanced via the formation of $\mathrm{H}_{2}$ molecules on the surface of dust grain (Hirashita \& Ferrara 2002; Cazaux \& Spaans 2004). In addition, the cooling of gas by thermal radiation from dust themselves makes even the gas clouds with metallicity as low as $10^{-5} Z_{\odot}$ fragment into low-mass gas clumps (Schneider et al. 2002, 2003; Omukai et al. 2005). Thus, dust

grains in the early universe may cause the characteristic mass scale of stars to shift from very high mass to low mass of $\sim 1 M_{\odot}$ observed at present time and strongly affect the evolution of the initial mass function (IMF) at the early epoch of the universe.

Also, dust grains in the early universe play crucial roles in revealing the structure and evolution of the universe from the relevant observations because they absorb stellar light and reemit it at infrared (IR)-submillimeter (submm) wavelengths. The thermal emission from dust at high redshifts can distort the cosmic microwave background (CMB) radiation (Loeb \& Haiman 1997), and the obscuration and reddening of starlight by dust residing in the early interstellar and/or intergalactic space lead to the serious underestimate in evaluating the cosmic SFR from the observations directed toward the higher redshifts (e.g., Hauser \& Dwek 2001).

In fact, the recent observations have confirmed the presence of large amounts of dust grains at high redshifts of $z \gtrsim 5$. The submm (Priddey et al. 2003; Robson et al. 2004) and millimeter (Bertoldi et al. 2003) observations reported continuum thermal emission of dust grains from some quasars at $z>5$, and have suggested a large amount of cold dust reaching up to $10^{8}-10^{9} M_{\odot}$ in the host galaxies of quasars. Maiolino et al. (2004a) discovered the substantial extinction of stellar light by dust in the broad absorption line (BAL) quasars at $z>4$.9. They have shown that their extinction curves could be different from those of low-redshift quasars at $z<4$, and have suggested that the difference may reflect different formation and evolution mechanisms of dust grains at $z \gtrsim 5$. 
The main sources of dust grains in the early universe at $z>5$ are considered to be supernovae ( $\mathrm{SNe}$ ) evolving from the massive stars because of their short lifetime. Dust grains formed in the ejecta of SNe are injected into the interstellar medium (ISM), and are subjected to destructive processes in the interstellar shocks induced by the ambient SNe (e.g., Jones et al. 1994; Dwek, Foster, \& Vancura 1996). Thus, the size distribution and amount of dust in the early interstellar space are determined by the balance between their production in SNe and their destruction in shocks, and change with time according to the star formation activity (e.g., Dwek 1998).

How much amount of dust grains are destructed in shocks as well as how much dust grains absorb stellar light and reemit it by thermal emission heavily depends on their chemical composition, size and amount. Thus, it is essential to clarify the evolution of dust in the early universe by treating the formation and destruction processes of dust grains in a consistent manner, in order to elucidate the SFR and the IMF during the early evolution of the universe from the future observations. Nozawa et al. (2003) investigated the chemical composition, size distribution and amount of dust grains formed in the ejecta of Pop III SNe. In this paper, as the second step to reveal the evolution of dust grains in the early universe, we explore dust destruction in the high-velocity interstellar shock driven by the SN explosion, adopting the dust model by Nozawa et al. (2003) for the initial dust residing in the early ISM.

We focus on dust destruction in nonradiative shock where dust grains are considered to be efficiently destructed by non-thermal and thermal sputtering (e.g., McKee 1989) because the nonradiative shock has a high shock velocity $\left(>100 \mathrm{~km} \mathrm{~s}^{-1}\right)$ and a high gas temperature $\left(>10^{6} \mathrm{~K}\right)$. However, only a few set of experimental data on sputtering yield are available for the projectile-target combinations of astrophysical interest. Hence, we first evaluate the sputtering yields for the combinations of dust species produced in Pop III SNe and ion species of interest to us, collecting a large amount of sputtering data and applying the universal relation for sputtering yield proposed by Bohdansky (1984) with a slight modification so as to reproduce well the experimental data. Then, we investigate the dependence of the efficiency of dust destruction for each grain species not only on the gas density $n_{\mathrm{H}, 0}$ in the ISM but also on the explosion energy $E_{51}$ and progenitor mass $M_{\mathrm{pr}}$ of SN driving the interstellar shocks by applying the hydrodynamical models of Pop III SNe by Umeda \& Nomoto (2002). Finally, we evaluate the time-scale of destruction for each grain species.

The efficiency of dust destruction by sputtering depends on the temperature and density of gas and the velocity of dust relative to gas in shock as well as the sputtering yield and the dust size. Thus, in the calculation, we solve the time evolution of temperature and density of gas in spherically symmetric shocks, including the cooling of gas by the atomic 
process, the inverse Compton scattering and the thermal emission of dust. In coupled with the hydrodynamical calculations for gas, we carefully treat the erosion of dust by thermal and non-thermal sputtering caused by motion of dust relative to gas, taking into account the size distribution of each dust species.

This paper is organized as follows: We first define the efficiency of dust destruction in $\S 2$. In $\S 3$, we describe the properties of the ISM in the early universe together with the dust model adopted in the calculations, and in $\S 4$, we present the basic equations of the hydrodynamic calculations for gas and the method of the simulation for dust destruction in shock. The sputtering yield of each grain species necessary for the calculations of dust destruction is evaluated in $\S 5$, and the grain physics in shock is described in $\S 6$. The results of calculations of dust destruction are given in details for the model of interstellar shock that is driven by a SN with $E_{51}=1$ and $M_{\mathrm{pr}}=20 M_{\odot}$ and is propagating into the ISM with $n_{\mathrm{H}, 0}=1 \mathrm{~cm}^{-3}$, and the approximation formula for the time-scale of dust destruction as a function of $E_{51}$ and $n_{\mathrm{H}, 0}$ is derived in $\S 7$, where the effects of the cooling of gas on the evolution of nonradiative shock and the destruction of dust are briefly discussed. The summary is presented in $\S 8$.

\section{The definition of the efficiency of dust destruction}

Our main goal is to reveal the time-scale of dust destruction by interstellar shocks in the early universe. In general, the time-scale of destruction $\tau_{\mathrm{SN}, j}$ for grain species $j$ is defined as (e.g., McKee 1989)

$$
\tau_{\mathrm{SN}, j}^{-1}=\epsilon_{j} \frac{M_{\mathrm{swept}}}{M_{\mathrm{ISM}}} \gamma_{\mathrm{SN}}
$$

where $\epsilon_{j}$ is the efficiency of destruction of grain species $j, M_{\text {swept }}$ is the mass of gas swept up by shock until the shock velocity $V_{\text {shock }}$ decelerates below $100 \mathrm{~km} \mathrm{~s}^{-1}, M_{\text {ISM }}$ is the mass of gas and dust in the ISM, and $\gamma_{\mathrm{SN}}$ is the effective SN rate. McKee (1989) has estimated $\epsilon_{j}$ to be nearly constant and considered that $M_{\text {swept }}$ is proportional to the SN explosion energy $E_{51}$ in units of $10^{51} \mathrm{erg}$. However, it is expected that both $\epsilon_{j}$ and $M_{\text {swept }}$ depend on not only $E_{51}$ but also the progenitor mass $M_{\mathrm{pr}}$ of SN and the number density of gas $n_{\mathrm{H}, 0}$ in the ISM. Hence, we aim at deriving the dependences of $\epsilon_{j}$ and $M_{\text {swept }}$ on $E_{51}, M_{\mathrm{pr}}$ and $n_{\mathrm{H}, 0}$. The

truncation time $t_{\text {tr }}$ being defined as the time at which $V_{\text {shock }}$ drops down to $100 \mathrm{~km} \mathrm{~s}^{-1}$, the efficiency of destruction $\epsilon_{j}$ for each dust species is calculated by

$$
\epsilon_{j}=\frac{M_{\mathrm{d}, j}^{\mathrm{dest}}\left(t=t_{\mathrm{tr}}\right)}{M_{\mathrm{d}, j}^{\mathrm{swept}}\left(t=t_{\mathrm{tr}}\right)},
$$


where $M_{\mathrm{d}, j}^{\text {dest }}(t)$ and $M_{\mathrm{d}, j}^{\text {swept }}(t)$ are, respectively, the mass of dust destructed and the mass of dust swept up by shock, and the mass of gas $M_{\text {swept }}$ swept up by spherically symmetric shock is given by

$$
M_{\text {swept }} \simeq \frac{4 \pi}{3} \rho_{0} R_{\text {shock }}^{3}\left(t=t_{\text {tr }}\right),
$$

with the gas density $\rho_{0}$ in the ISM and the travel distance of the shock front $R_{\text {shock }}(t)$.

\section{Properties of the ISM in the early universe}

\subsection{Density, temperature, and metallicity of the ISM}

We assume that the ISM in the early universe is homogeneous and stationary $(v=0)$, referring to the results of the simulations on the formation of the early H II region by the first stars (Kitayama et al. 2004), which have shown that the radiative feedback from the massive stars causes the ambient ISM to be homogenized with the mean gas density of $\lesssim$ $1 \mathrm{~cm}^{-3}$. In the calculations, we consider the hydrogen number density in the ISM to be in the range of $0.03 \mathrm{~cm}^{-3} \leq n_{\mathrm{H}, 0} \leq 10 \mathrm{~cm}^{-3}$ to reveal the dependence of the efficiency of dust destruction on the gas density in the ISM. The ISM surrounding the SN explosion is considered to be ionized by the radiation of the massive progenitor stars and heated to by $T_{0} \sim 10^{4} \mathrm{~K}$ (Kitayama et al. 2004; Machida et al. 2005), and then $T_{0}$ is expected to decrease below $10^{4} \mathrm{~K}$ where the recombination rate can be high even for the metallicity as low as $Z=10^{-4} Z_{\odot}$ (Wolfire et al. 1995). However, the evolution of gas temperature in postshock flow is almost independent of $T_{0}$ until the end of the Sedov-Taylor phase of high-velocity shock considered in this paper, and the temperature of gas $T_{\text {shock }}$ at the shock front does not decrease below several of $10^{4} \mathrm{~K}$ at the truncation time, as is shown in the result of calculations (see 7.1). Thus, we take $T_{0}=10^{4} \mathrm{~K}$ as the representative gas temperature in the ISM, independently of the density of gas.

The truncation time as well as the time evolution of gas temperature and density structures in postshock flow is considered to be affected by the cooling of gas depending on the metallicity of the ISM. Especially, when $T_{\text {shock }} \lesssim 10^{5} \mathrm{~K}$, the efficient line coolings of $\mathrm{H}$ and He ions cause the shock velocity to decelerate rapidly, and resultingly regulate the truncation time. However, the gas cooling function is almost independent of the metallicity for $Z \lesssim 10^{-3} Z_{\odot}$ corresponding to $[\mathrm{Fe} / \mathrm{H}] \leq-3$ since the metal lines do not contribute to the cooling of gas at $T \geq 10^{4} \mathrm{~K}$ (Sutherland \& Dopita 1993), and we adopt the gas cooling function for the zero metal case given in Sutherland \& Dopita (1993) (see 4.1). Note that the efficiency of dust destruction, which is defined by the mass ratio of dust destructed to 
dust swept up by shock, does not depend on the metallicity as long as $Z \lesssim 10^{-3} Z_{\odot}$, despite the fact that the amount of dust in the ISM is proportional to the metallicity, and in the calculations we assume that the metallicity of the ISM in the early universe is $Z=10^{-4}$ $Z_{\odot}$ to investigate the destruction of dust in the ISM. The elemental composition of gas in the ISM is given in Table 1 where the $\mathrm{C}, \mathrm{N}$, and $\mathrm{O}$ metals are included so as to adjust the metallicity to $Z=10^{-4} Z_{\odot}$ and their abundances are simply evaluated by multiplying the metallicity and the primordial ratios tabulated in Sutherland \& Dopita (1993); note that these metals play no role in the physical processes relevant to dust destruction because of the much lower abundances than $\mathrm{H}$ and He. The electron number density $n_{e}$ is fixed to 1.128 $n_{\mathrm{H}}$ given by Sutherland \& Dopita (1993).

\subsection{Model of dust in the early universe}

Dust grains in the ISM at the early epoch of the universe considered in this paper are expected to be predominantly produced in the ejecta of SNe. To reveal what kind of dust grains form in the early universe, Todini \& Ferrara (2001) calculated dust formation in primordial core-collapse supernovae (CCSNe), assuming the uniform elemental composition and gas density within the He core. They have shown that the newly formed grains are amorphous carbon with size around $300 \AA$, and $\mathrm{Al}_{2} \mathrm{O}_{3}, \mathrm{MgSiO}_{3}, \mathrm{Mg}_{2} \mathrm{SiO}_{4}$ and $\mathrm{Fe}_{3} \mathrm{O}_{4}$ whose sizes are around 10-20 $\AA$. By extending the model of dust formation by Todini \& Ferrara (2001) to pair-instability supernovae (PISNe) whose progenitors evolve from metal-free stars with $M_{\mathrm{pr}}=140-260 M_{\odot}$ (Umeda \& Nomoto 2002; Heger \& Woosley 2002), Schneider, Ferrara, \& Salvattera (2004) found that the typical sizes of dust formed in PISNe range from 0.001 to $0.3 \mu \mathrm{m}$ depending on grain species. The dust models by Todini \& Ferrara (2001) can successfully reproduce the extinction curve observed toward a BAL quasar SDSS1048+46 at $z=6.2$ (Maiolino et al. 2004b) and the IR spectral energy distribution (SED) of blue compact dwarf galaxy SBS 0335-052 (Takeuchi et al. 2003) except for the slight overestimate of the far IR continuum; SBS 0335-052 is a good target in the local universe suitable for scrutinizing the properties of dust formed in SNe at high redshifts because of its young age $\left(<10^{7} \mathrm{yr}\right)$ and low metallicity $\left(1 / 41 Z_{\odot}\right)$ (Vanzi et al. 2000).

However, as discussed by Kozasa, Hasegawa, \& Nomoto (1989), the species of dust formed in the ejecta largely depend on the elemental composition in the He core, and their sizes are affected by the time evolution of gas density and temperature. Thus, Nozawa et al. (2003) extensively investigated dust production in Pop III CCSNe and PISNe, by considering two extreme cases for the elemental composition in the ejecta; the unmixed case with the original onion-like structure and the uniformly mixed case within the He core. In 
the calculations of dust formation, the radial profile of gas density is properly treated and the time evolution of gas temperature is calculated by solving the radiative transfer equation including the energy deposition of radioactive elements, which are not taken into account by Todini \& Ferrara (2001). Nozawa et al. (2003) found that a variety of grain species (C, Si, $\mathrm{Fe}, \mathrm{FeS}, \mathrm{Al}_{2} \mathrm{O}_{3}, \mathrm{MgSiO}_{3}, \mathrm{Mg}_{2} \mathrm{SiO}_{4}, \mathrm{SiO}_{2}$ and $\mathrm{MgO}$ ) condense in the unmixed ejecta reflecting the difference of the elemental composition at the formation site, and that only oxide and silicate grains $\left(\mathrm{Al}_{2} \mathrm{O}_{3}, \mathrm{MgSiO}_{3}, \mathrm{Mg}_{2} \mathrm{SiO}_{4}, \mathrm{SiO}_{2}\right.$ and $\left.\mathrm{Fe}_{3} \mathrm{O}_{4}\right)$ form in the mixed ejecta with $\mathrm{C} / \mathrm{O}<1$. The average sizes of these grains span the range of $0.001-1 \mu \mathrm{m}$ depending on the elemental composition and gas density at the location of formation in the ejecta. The species of dust formed in Pop III SNe and the behaviors of their size distributions are almost independent of the progenitor mass, but the relative mass fraction of each grain species is dependent on.

Based on the dust models by Nozawa et al. (2003), Hirashita et al. (2005) have shown that the extinction curve of a quasar SDSS1048+46 at $z=6.2$ can be better explained by the dust grains produced in the unmixed Pop III CCSNe than those in the mixed Pop III CCSNe. Takeuchi et al. (2005) have also shown by using the dust models for $M_{\mathrm{pr}}=20 M_{\odot}$ that only dust grains formed in the unmixed ejecta but not in the mixed ejecta can give an excellent fit to the observed SED of SBS 0335-052 over near to far IR wavelengths. These works strongly suggest that the comparisons with the observations relevant to dust in the early universe prefer dust grains produced in the unmixed Pop III CCSNe rather than those in the mixed Pop III CCSNe.

Therefore, as the representative model of the initial dust grains residing in the early ISM, we adopt the size distribution and abundance of each grain species obtained by the calculations of dust formation in the unmixed ejecta of Pop III SN with $M_{\mathrm{pr}}=20 M_{\odot}$ by Nozawa et al. (2003). For comparison, we also consider the model of dust formed in the mixed ejecta of the same SN. Hereafter, the models of dust formed in the unmixed and mixed ejecta are referred to as the unmixed grain model and the mixed grain model, respectively. The initial values of the depletion factor defined by the ratio of the total dust mass to the total metal mass produced in the SN is 0.224 for the unmixed grain model and 0.297 for the mixed grain model. The bulk density $\rho_{\mathrm{d}, j}$, mass fraction $A_{j}$ and average size $a_{\mathrm{ave}, j}$ of each grain species are summarized in Table 2, and their size spectrums are given in Figure 6a for the unmixed grain model and in Figure 7a for the mixed grain model, where the bin width is 0.1 dex.

It is useful for the following discussion to address here the behavior of size distribution of each dust species produced in the unmixed ejecta. $\mathrm{C}, \mathrm{Fe}$ and $\mathrm{SiO}_{2}$ grains have a lognormallike size distribution with the relatively large average radius of $>0.01 \mu \mathrm{m}$, while the size 
distribution functions of $\mathrm{FeS}, \mathrm{Mg}_{2} \mathrm{SiO}_{4}$ and $\mathrm{MgO}$ grains are approximately power-law. The average radii of $\mathrm{Al}_{2} \mathrm{O}_{3}$ and $\mathrm{MgSiO}_{3}$ grains are very small $\left(<5 \times 10^{-3} \mu \mathrm{m}\right)$. Note that $\mathrm{Si}$ grain shows a bimodal size distribution; the grains with the size less than $0.03 \mu \mathrm{m}$ occupy almost $100 \%$ in number but only $0.2 \%$ in mass. Thus, as the average radius of Si grain, we take $0.25 \mu \mathrm{m}$ calculated for the grains larger than $0.03 \mu \mathrm{m}$ including $99.8 \%$ in mass. The size distribution function summed up over all grain species is well fitted with a broken power-law formula whose index is -3.5 for the radius larger than $0.06 \mu \mathrm{m}$ and -2.5 for the smaller one.

\section{Physics of interstellar shock}

The deceleration rate due to drag force and the erosion rate due to sputtering of dust grains depend on the time evolution of gas temperature and density in postshock flow. In this section, we present the basic equations and initial conditions for the hydrodynamical cal-

culation of the time evolution of shock and the method of the simulation for dust destruction in shock.

\subsection{The basic equations for the gas dynamics}

The hydrodynamic equations for gas to calculate the time evolution of spherically symmetric interstellar shock driven by a SN explosion, using conventional physical variables, are given by

$$
\begin{aligned}
\frac{\partial \rho}{d t}+\frac{1}{r^{2}} \frac{\partial}{\partial r}\left(r^{2} \rho v\right) & =0 \\
\frac{\partial}{d t}(\rho v)+\frac{1}{r^{2}} \frac{\partial}{\partial r}\left(r^{2} \rho v^{2}\right) & =-\frac{\partial P}{\partial r} \\
\frac{\partial U}{d t}+\frac{1}{r^{2}} \frac{\partial}{\partial r}\left[r^{2}(U+P) v\right] & =-\Lambda\left(n_{\mathrm{H}}, T\right),
\end{aligned}
$$

where $P=\rho k T / \mu m_{\mathrm{H}}$ is the gas pressure with the mean molecular weight $\mu$ and the hydrogen mass $m_{\mathrm{H}}$, and the sum of kinetic energy and thermal energy per unit volume is

$$
U=\frac{1}{2} \rho v^{2}+\frac{P}{\gamma-1}
$$

with adiabatic index $\gamma$, and we adopt $\gamma=5 / 3$ in the calculation. 
The cooling rate of gas $\Lambda\left(n_{\mathrm{H}}, T\right)$ in units of $\mathrm{erg} \mathrm{s}^{-1} \mathrm{~cm}^{-3}$ is expressed by

$$
\Lambda\left(n_{\mathrm{H}}, T\right)=n_{e} n_{\mathrm{H}} \Lambda_{\text {gas }}(T)+\Lambda_{\mathrm{ic}}(T)+\Lambda_{\mathrm{d}}\left(n_{\mathrm{H}}, T\right),
$$

where each term represents the rate of cooling by the atomic process, by the inverse Compton scattering, and by the thermal emission of dust. In the calculations, we adopt the gas cooling function $\Lambda_{\text {gas }}(T)$ for the zero metal case given in Sutherland \& Dopita (1993) and ignore the contribution of cooling by metal ions ejected from dust by sputtering, because as mentioned in 3.1, the metals do not contribute to the cooling of gas for the metallicity of $Z=10^{-4}$ $Z_{\odot}$ (Sutherland \& Dopita 1993). For the inverse Compton scattering caused by collisions of hot electrons in shock with CMB photons, the cooling rate $\Lambda_{\text {ic }}(T)$ is given by (Ikeuchi \& Ostriker 1986)

$$
\Lambda_{\mathrm{ic}}(T)=5.41 \times 10^{-32}(1+z)^{4} n_{e} T_{4}
$$

where $T_{4}$ is the gas temperature in units of $10^{4} \mathrm{~K}$, and we take $z=20$ in the calculation. The cooling function $\Lambda_{\mathrm{d}}\left(n_{\mathrm{H}}, T\right)$ through the thermal emission from dust heated by collisions with gas particles in shock is calculated by (Dwek 1987)

$$
\Lambda_{\mathrm{d}}\left(n_{\mathrm{H}}, T\right)=\sum_{j} n_{\mathrm{d}, j} \int H_{j}\left(a, T, n_{\mathrm{H}}\right) f_{j}(a) d a,
$$

where $n_{\mathrm{d}, j}$ and $f_{j}(a)$ are, respectively, the number density and size distribution function of dust species $j$ at a given $t$ and $r$. The collisional heating rate $H_{j}\left(a, T, n_{\mathrm{H}}\right)$ of grains species $j$ is given in 6.3 .

The time evolution of gas temperature and density in shock driven by SN is characterized by the explosion energy $E_{51}$ and the progenitor mass $M_{\mathrm{pr}}$ which specify the structures of density and velocity of gas in the ejecta. Thus, as the initial condition for interstellar shocks, we adopt the hydrodynamical models of the ejecta of Pop III SNe with various $E_{51}$ and $M_{\mathrm{pr}}$ by Umeda \& Nomoto (2002) to investigate the dependence of the efficiency of dust destruction on $E_{51}$ and $M_{\mathrm{pr}}$. Note that in the early universe with $Z=10^{-4} Z_{\odot}$, the progenitors of SNe are not only Pop III stars but also massive stars of the subsequent generation. However, the structures of density and velocity in the SN with a given $E_{51}$ and $M_{\mathrm{pr}}$ do not so much depend on the initial metallicity for the progenitor star whose metallicity is less than $10^{-4}$ $Z_{\odot}$ (Umeda, H. 2002 in private communication). In the calculations, we consider ordinary CCSNe with the explosion energy of $E_{51}=1$, hypernovae (HNe) whose explosion energy is more than ten times that of CCSNe, and PISNe whose progenitor is very massive $\left(M_{\mathrm{pr}}=150\right.$, 170 and $200 M_{\odot}$ ), and hereafter CCSNe and HNe with progenitor mass of $M_{\mathrm{pr}}=13-30 M_{\odot}$ are referred to as Type II SNe (SNe II). The ejecta models used in the calculations are given in Table 3, where the labels $\mathrm{C}, \mathrm{H}$ and $\mathrm{P}$ represent CCSNe, HNe and PISNe, respectively, and the numerical value denotes the mass of the progenitor in units of solar mass. 


\subsection{The method of simulations}

Equations (4)-(6) are solved by using the flux-splitting method with second-order accuracy in space and first-order accuracy in time (van Albada, van Leer, \& Roberts 1982; Mair et al. 1988). This algorithm is one of the upwind schemes for the Euler equations and is well suited to solve problems involving a shock.

The calculations for the time evolution of shock and dust destruction are performed via the following two steps. In the first step, we assume that the SN ejecta collides with the ISM in ten years after the explosion and calculate the time evolution of gas density and temperature without including dust, based on the ejecta models of Umeda \& Nomoto (2002). The spatial mesh number is 500-5000 depending on the gas density in the ISM, and the inner 20 meshes are assigned to the ejecta. Note that dust destruction in this first step does not contributes to the evaluation of the destruction efficiency because the mass of gas swept up by shock is less than $0.1 \%$ of that by $t=t_{\mathrm{tr}}$. Also, the cooling of gas by dust has no effects on the subsequent structure of shock. In the second step, fixing the spatial mesh number to 430 , we rearrange the density, temperature and velocity of postshock gas obtained from the first step into the inner 30 linear meshes. Then, including dust, we calculate the time evolution of shock as well as dynamics and destruction of dust.

By treating dust as a test particle, the physical processes relevant to dust are calculated as follows. Consider a stationary $\left(v_{\mathrm{d}}=0\right)$ dust grain characterized by its composition and number density with radius between $a$ and $a+d a$ at a given position $R_{\mathrm{d}}$ in the ISM. Once the grain enters into the shock front, the relative velocity to gas $w_{\mathrm{d}}=v-v_{\mathrm{d}}$ is assigned. Then, for the temperature $T$ and density $n_{\mathrm{H}}$ of gas at the shock front, we calculate the deceleration rate $d w_{\mathrm{d}} / d t$ due to drag force, the erosion rate $d a / d t$ by sputtering and the heating rate $H\left(a, T, n_{\mathrm{H}}\right)$ by collisions with gas. By updating the relative velocity by $w_{\mathrm{d}}^{\prime}=w_{\mathrm{d}}+\left(d w_{\mathrm{d}} / d t\right) \Delta t$ and the grain radius by $a^{\prime}=a+(d a / d t) \Delta t$ for a given small time step $\Delta t$, the velocity and position of the dust at time $t^{\prime}=t+\Delta t$ are calculated by $v_{d}^{\prime}=v+w_{d}^{\prime}$ and $R_{\mathrm{d}}^{\prime}=R_{\mathrm{d}}+v_{\mathrm{d}}^{\prime} \Delta t$. The procedure described above is repeated for the gas temperature $T$ and density $n_{\mathrm{H}}$ at the position $R_{\mathrm{d}}^{\prime}$ of the grain. Note that the dust grain is considered to be completely destroyed if the grain radius is smaller than the nominal monomer radius of condensate given in Nozawa et al. (2003).

\section{Evaluation of sputtering yield}

Sputtering is divided into two categories reflecting the difference in the underlying mechanisms; physical sputtering and chemical sputtering. Physical sputtering invokes a transfer 
of kinetic energy from the incident particle to target atoms and subsequent ejection of those atoms which have acquired kinetic energy enough to overcome the binding forces exerted by the target. Chemical sputtering invokes a chemical reaction induced by the impinging particles which produces an unstable compound at the target surface (Sigmund 1981). In the high-velocity shocks considered in this paper, dust grains are predominantly destroyed by physical sputtering because dust temperature is at most $100 \mathrm{~K}$ in the postshock region (Dwek 1987); chemical sputtering is of no importance since this process is realized at the temperature as high as $800 \mathrm{~K}$ (Roth 1983). Thus, in what follows, we simply refer to physical sputtering as "sputtering". The efficiency of destruction by sputtering is characterized by the sputtering yield defined as the mean number of emitted atoms per incident particle. The sputtering yield depends on the impact energy of a projectile and the incident angle to the target surface as well as on the projectile-target combination. However, only a few set of experimental data on sputtering yield are available for the combinations of dust materials and ion species of interest to us.

One method evaluating the sputtering yields for the projectile-target combinations of astrophysical interest is to apply the Monte-Carlo code for the TRANSPORT OF IONS IN MATTER (TRIM, Ziegler, Biersack, \& Littmark 1985) that simulates the energy loss of a projectile ion with given impact energy and incident angle in a solid (Field et al. 1997; May et al. 2000; Bianchi \& Ferrara 2005). The TRIM code requires as input parameters not only the surface binding energy of the target, but also the displacement energy $E_{\mathrm{d}}$ and the lattice binding energy $E_{\mathrm{b}}$. Particularly, uncertainties in the value of $E_{\mathrm{d}}$ are a significant source of error in the calculated sputtering yield (May et al. 2000; Bianchi \& Ferrara 2005). In fact, the sputtering yields computed by this simulation code have been known to significantly differ from the experimental data at low impact energies (Bianchi \& Ferrara 2005). Furthermore, it is time-consuming to combine the Monte-Carlo simulation with the hydrodynamical calculation for dust destruction.

Another one is to apply the universal relation which is the analytic formula derived by Bohdansky (1984) for the energy dependence of sputtering yield at normal incidence for a monatomic solid. To investigate the destruction of interstellar dust such as $\mathrm{C}, \mathrm{Fe}, \mathrm{SiC}, \mathrm{SiO}_{2}$ and $\mathrm{H}_{2} \mathrm{O}$ ice, Tielens et al. (1994) have applied the universal relation by treating a constant $K$ in this formula as a parameter, and determined it by comparing the available experimental data with the sputtering yield calculated by the universal relation. They have shown that the universal relation can be applied to not only monatomic solids but also compound solids by taking the appropriate average values for the atomic and mass numbers and the surface binding energy under the assumption that each of element consisting of the target is sputtered off with the same rate. However, the agreement of the calculated sputtering yield with the experimental data is not so much good for the ion-target combinations with the mass ratio 
of target atom to incident ion ranging from 0.7 and 2 .

Therefore, we apply the universal relation for sputtering yield with a slight modification so as to reproduce well the experimental data, collecting and examining minutely a large amount of sputtering data, in order to evaluate the sputtering yields for the combinations of dust and ion species of interest to us. Following Tielens et al. (1994), we treat $K$ as a free parameter and determine it by fitting to the experimental data for the grain species with the yield data covering a wide range of impact energy of the projectiles. For the grain species for which little or no sputtering data are available, we deduce the value of $K$ by fitting to the sputtering yield calculated by means of the Monte-Carlo code for the EROSION AND DEPOSITION BASED ON DYNAMIC MODEL (EDDY, Hirooka 2001). The EDDY code requires only the surface binding energy as an input parameter and allows us to predict the sputtering yield for specific impact energy and incident angle more easily than the TRIM code, though the energy range applicable to the calculations is limited to $0.1-10 \mathrm{keV}$. In the following subsection, we at first introduce the universal relation for sputtering yield, and then determine the value of $K$ for each grain species by fitting to the available experimental data and/or the results of the EDDY simulation.

\subsection{Universal relation for sputtering yield}

For backward sputtering and with the neglect of inelastic energy losses, the sputtering yield at normal incidence $Y_{i}^{0}(E)$ by the projectile $i$ impacting with energy $E$ is given by (Bohdansky 1984)

$$
Y_{i}^{0}(E)=4.2 \times 10^{14} \frac{S_{i}(E)}{U_{0}} \frac{\alpha_{i}\left(\mu_{i}\right)}{K \mu_{i}+1}\left[1-\left(\frac{E_{\mathrm{th}}}{E}\right)^{2 / 3}\right]\left(1-\frac{E_{\mathrm{th}}}{E}\right)^{2},
$$

where $U_{0}$ is the surface binding energy in units of $\mathrm{eV}, \mu_{i}=M_{\mathrm{d}} / M_{i}$ is the ratio of the mass number of the target atom to the incident ion, $\alpha_{i}$ is the energy-independent function of $\mu_{i}$, and $K$ is considered to be a free parameter to be adjusted to reproduce the experimental data (Tielens et al. 1994). The threshold energies $E_{\text {th }}$ have been obtained by fitting the yield data for low-energy sputtering and are approximately given by (Bohdansky et al. 1980; Andersen \& Bay 1981)

$$
\begin{array}{ll}
E_{\mathrm{th}}=\frac{U_{0}}{g_{i}\left(1-g_{i}\right)} & \text { for } \frac{M_{i}}{M_{\mathrm{d}}} \leq 0.3 \\
E_{\mathrm{th}}=8 U_{0}\left(\frac{M_{i}}{M_{\mathrm{d}}}\right)^{1 / 3} & \text { for } \quad \frac{M_{i}}{M_{\mathrm{d}}}>0.3,
\end{array}
$$


where $g_{i}=4 M_{i} M_{\mathrm{d}} /\left(M_{i}+M_{\mathrm{d}}\right)^{2}$ is the maximum fractional energy transfer possible in a head-on elastic collision. The function $S_{i}(E)$ is the nuclear stopping cross section in units of ergs $\mathrm{cm}^{2}$ and can be expressed by the following universal relation (Sigmund 1981)

$$
S_{i}(E)=4 \pi a_{\mathrm{sc}} Z_{i} Z_{\mathrm{d}} e^{2} \frac{M_{i}}{M_{i}+M_{\mathrm{d}}} s_{i}\left(\epsilon_{i}\right)
$$

where $e$ is the elementary charge, and $Z_{i}$ and $Z_{\mathrm{d}}$ are the atomic numbers of the projectile and the target, respectively. The screening length $a_{\mathrm{sc}}$ for the interaction potential between the nuclei is given by

$$
a_{\mathrm{sc}}=0.885 a_{0}\left(Z_{i}^{2 / 3}+Z_{\mathrm{d}}^{2 / 3}\right)^{-1 / 2}
$$

with the Bohr radius $a_{0}=0.529 \AA$. The function $s_{i}\left(\epsilon_{i}\right)$ depends on the detailed form adopted for the screened Coulomb interaction and can be approximated by (Matsunami et al. 1980)

$$
s_{i}\left(\epsilon_{i}\right)=\frac{3.441 \sqrt{\epsilon_{i}} \ln \left(\epsilon_{i}+2.718\right)}{1+6.35 \sqrt{\epsilon_{i}}+\epsilon_{i}\left(-1.708+6.882 \sqrt{\epsilon_{i}}\right)},
$$

where the reduced energy $\epsilon_{i}$ is given by

$$
\epsilon_{i}=\frac{M_{\mathrm{d}}}{M_{i}+M_{\mathrm{d}}} \frac{a_{\mathrm{sc}}}{Z_{i} Z_{\mathrm{d}} e^{2}} E .
$$

The value of $\alpha_{i}\left(\mu_{i}\right)$ in Equation (11) depends on how the distribution of energy deposited in the target is approximated. Sigmund (1969) suggested the three types of approximations according to the adopted distribution function (Gaussian, corrected Gaussian, and nonGaussian). The values of $\alpha_{i}\left(\mu_{i}\right)$ obtained by these approximations are very similar (Sigmund 1969) and is approximately expressed for $\mu_{i} \leq 10$ as follows (e.g., Bohdansky 1984);

$$
\begin{array}{ll}
\alpha_{i}=0.2 & \text { for } \mu_{i} \leq 0.5 \\
\alpha_{i}=0.3 \mu_{i}^{2 / 3} & \text { for } 0.5<\mu_{i} \leq 10
\end{array}
$$

However, Equation (17) for $\alpha_{i}$ slightly overestimates the sputtering yields for the projectiletarget combinations with the mass ratio of $0.7 \lesssim \mu_{i} \lesssim 2$. Hence, from a comparison with a large amount of sputtering data, we apply the following formula for $\alpha_{i}$;

$$
\begin{array}{ll}
\alpha_{i}=0.2 & \text { for } \mu_{i} \leq 0.5 \\
\alpha_{i}=0.1 \mu_{i}^{-1}+0.25\left(\mu_{i}-0.5\right)^{2} & \text { for } 0.5<\mu_{i} \leq 1 \\
\alpha_{i}=0.3\left(\mu_{i}-0.6\right)^{2 / 3} & \text { for } 1<\mu_{i} .
\end{array}
$$


As we shall show below, this modified function $\alpha_{i}$ can give an excellent agreement with the sputtering data for any values of $\mu_{i}$ considered here $\left(0.3 \leq \mu_{i} \leq 56\right)$.

By being $K$ as a parameter, the surface binding energy $U_{0}$ must be specified to evaluate the normal-incidence sputtering yield $Y_{i}^{0}(E)$ from Equation (11). We assume $U_{0}$ to be equal to the sublimation energy and evaluate it from the JANAF Thermochemical Tables (Chase et al. 1985) except for $\mathrm{C}$ and $\mathrm{Al}_{2} \mathrm{O}_{3}$ whose values of $U_{0}$ are discussed later. The values of $U_{0}$ for each grain species are summarized in Table 4 along with the values of $Z_{\mathrm{d}}$ and $M_{\mathrm{d}}$.

\subsection{Sputtering yield of each grain species}

In order to evaluate the sputtering yield for the projectile-target combinations of interest to us, we determine the value of $K$ by fitting to the experimental data for $\mathrm{C}, \mathrm{Si}, \mathrm{Fe}, \mathrm{SiO}_{2}$ and $\mathrm{Al}_{2} \mathrm{O}_{3}$ grains for which a sufficient amount of sputtering data are available. For $\mathrm{MgO}$, $\mathrm{FeS}$ and $\mathrm{Fe}_{3} \mathrm{O}_{4}$ grains for which little or no yield data are available, we evaluate the value of $K$ by fitting to the results of the EDDY simulation. The experimental and simulated sputtering data at normal incidence, for which the references are summarized in Table 4, are given in Figure 1 along with the best-fitting theoretical yield calculated by the universal relation (solid lines). In Figures $1 \mathrm{a}-1 \mathrm{e}$, the experimental data are shown for the following ion species; $\mathrm{H}^{+}$(open circles), $\mathrm{D}^{+}$(asterisks), $\mathrm{He}^{+}$(open squares), $\mathrm{Ne}^{+}$(crosses) and $\mathrm{Ar}^{+}$ (open triangles). Even in the case that the experimental data can be sufficiently available, for comparison, we also plot the results of the EDDY calculations for the projectiles $\mathrm{H}^{+}$ (filled circles), $\mathrm{He}^{+}$(filled squares) and $\mathrm{Ar}^{+}$(filled triangles).

As for $\mathrm{C}$, the sublimation energy estimated to be $U_{0}=7.43 \mathrm{eV}$ from the thermodynamical data cannot reproduce the experimental data. Tielens et al. (1994) have shown that the ion fluences efficiently amorphize the surface layer of carbon material and reduce the surface binding energy. Thus, we take $U_{0}=4.0 \mathrm{eV}$ following Tielens et al. (1994), and this value gives a good fit to the measured yields with $K=0.61$ (Fig. 1a) being different from $K=0.65$ by Tielens et al. (1994); the difference stems from the adopted formula for $\alpha_{i}$. The sputtering yield calculated by the EDDY code is enhanced for $\mathrm{H}^{+}$by a couple of factor compared to the experimental data, but gives a good agreement for $\mathrm{He}^{+}$.

The sputtering data for Si and Fe targets are sufficiently available. The modified universal relation employing Equation (18) for $\alpha_{i}$ shows the excellent agreement with the data points for all projectiles considered here (Figs. $1 \mathrm{~b}$ and 1c), by adopting $K=0.43$ and $K=0.23$, respectively. The EDDY calculations for $\mathrm{Si}$ and Fe targets also present a good agreement with their measured data. The dotted lines indicate the sputtering yields by 
$\mathrm{Ar}^{+}$projectile calculated by using the values of $K$ derived above but adopting $\alpha_{i}$ given by Equation (17). It can be seen from these figures that Equation (17) overestimates the sputtering yields for the projectile-target combinations with the mass ratio of $0.7 \lesssim \mu_{i} \lesssim 2$ as mentioned in 5.1. It should be emphasized that the previous formula cannot reproduce the experimental data better than the modified universal relation whatever values of $K$ are selected.

A very good fit of the universal relation to the experimental data is obtained for $\mathrm{SiO}_{2}$ target by taking $K=0.1$ (Fig. 1d). This justifies the application of the universal relation to compound materials as shown by Tielens et al. (1994). The results by the EDDY simulations are consistent with the experimental data, but a comparison with the theoretical curves of sputtering yield shows a little enhancement for $\mathrm{H}^{+}$and $\mathrm{Ar}^{+}$projectile at $E \leq 200 \mathrm{eV}$. For $\mathrm{MgSiO}_{3}$ and $\mathrm{Mg}_{2} \mathrm{SiO}_{4}$ materials for which the sputtering data cannot be found in the literatures, as is the same as Tielens et al. (1994), we assume $K$ to be 0.1, considering $\mathrm{SiO}_{2}$ as a representative of silicates. In fact, this could be reasonable since $K \simeq 0.1$ for the compounds including oxygen atoms (see Table 4).

Given the surface binding energy of $U_{0}=6.37 \mathrm{eV}$ evaluated from the thermodynamical data, the yield data of $\mathrm{Al}_{2} \mathrm{O}_{3}$ cannot be reproduced by the universal relation for any values of $K$. The disagreement of the theoretical prediction with the yield data is expected to be due to an unsuitable estimate of $U_{0}$, as is the same as C. Indeed, Roth, Bohdansky, \& Ottenberger (1979) have suggested $U_{0}=8.5 \mathrm{eV}$ for $\mathrm{Al}_{2} \mathrm{O}_{3}$ from the behavior of sputtering yield at impact energies below the maximum yield by $\mathrm{He}^{+}$. Adopting this surface binding energy, we can get a better fit with $K=0.08$ (Fig. 1e), although the agreement of the universal relation with the yield data is the worst among grain materials considered in this paper.

To our knowledge, little or no experimental data on sputtering yield exist for $\mathrm{MgO}, \mathrm{FeS}$ and $\mathrm{Fe}_{3} \mathrm{O}_{4}$. Thus, we employ the results by the EDDY calculations to extract the values of $K$ for these materials. In the figures from $1 \mathrm{f}$ to $1 \mathrm{~h}$ (1f for $\mathrm{MgO}, 1 \mathrm{~g}$ for $\mathrm{FeS}$, and $1 \mathrm{~h}$ for $\mathrm{Fe}_{3} \mathrm{O}_{4}$ ), we show the EDDY results and the best-fitting theoretical curves. The agreements are not always good for $\mathrm{H}^{+}$and $\mathrm{Ar}^{+}$at low energies, which is also true for $\mathrm{SiO}_{2}$, but the universal relation well reproduces the yield data of $\mathrm{SiO}_{2}$. Thus, we consider that the fits are reasonable and satisfactory for these materials. The values of $K$ determined are 0.06, 0.18 and 0.15 for $\mathrm{MgO}, \mathrm{FeS}$ and $\mathrm{Fe}_{3} \mathrm{O}_{4}$, respectively.

Table 4 summarizes the value of $K$ for each grain species evaluated by fitting to the available experimental data and/or the results of the EDDY simulations. We confirm that the derived value of $K$ can well reproduce the experimental data of compounds as well as single-element materials, by adopting appropriate average values for the atomic and mass 
numbers as proposed by Tielens et al. (1994). By slightly modifying the function $\alpha_{i}\left(\mu_{i}\right)$ in the universal relation, we realized that the calculated sputtering yields well agree with the available experimental data than before. The modified universal relation derived here could be applicable to evaluate the sputtering yield for any combinations of targets and projectiles of astrophysical interest as long as the experimental data are available.

\section{Physics of dust grains in shock}

The collisional interactions between dust and a hot gas in shock efficiently transfers charge, momentum and energy between the two phases. As a result, dust grains acquire the electric charge, undergo the resistance to their motion through the gas, are eroded by sputtering, and are heated by collisions with gas. Also, heated dust grains cool the gas through their thermal emission.

Electric charge on a grain plays an important role in grain physics in hot plasmas through the Coulomb interaction and the Lorentz force in the presence of magnetic field. Here, we concentrate on the destruction of dust grains in fast $\left(\geq 100 \mathrm{~km} \mathrm{~s}^{-1}\right)$ and hot $\left(\geq 10^{5} \mathrm{~K}\right.$ ) nonradiative shocks. For $T \gtrsim 10^{6} \mathrm{~K}$, the effect of the electric charge of dust grain can be neglected because the dimensionless potential parameter $\phi$ defined by the ratio of the electric potential acquired by dust to the kinetic energy of gas is approximated by $\phi \sim 10^{5} / T$ and is significantly smaller than unity (Draine \& Salpeter 1979; McKee et al. 1987). Also, the erosion rate of refractory grains by thermal sputtering quickly decreases for

$T<10^{6} \mathrm{~K}$ (Draine \& Salpeter 1979). Correspondingly, the erosion rate of dust grans by non-thermal sputtering decreases quickly for the relative velocity of dust to gas $w_{\mathrm{d}}<200$ $\mathrm{km} \mathrm{s}^{-1}$. Therefore, it can be expected that the electric charge does not significantly affect the destruction of dust grains in nonradiative shocks considered in this paper. Also, it is assumed that the early ISM is not pervaded by a magnetic field because the magnetic field in the early universe is considered to be very weak (Gnedine, Ferrara, \& Zweibel 2000). Thus, we formulate the basic equations describing the motion of dust grains and their erosion by sputtering in postshock flow, neglecting the effect of charge on dust grain.

\subsection{Dynamics of dust grain}

Once dust grains being at rest in the preshock ISM encounter the interstellar shock, they move ballistically behind the shock front with the initial velocity relative to gas $w_{\mathrm{d}} \simeq$ $(3 / 4) V_{\text {shock }}$ where $V_{\text {shock }}$ is the shock velocity, and are eroded by non-thermal sputtering. 
A grain streaming through the ionized gas is decelerated by a drag force due to the direct collisions with gas particles, and thus the velocity of dust relative to gas decreases. The drag force acting on a grain results from the momentum transfer from gas to dust. Under the assumption that a gas particle reflects specularly from grain surface and that the kinetic temperature of gas is the same for all gas species, the deceleration rate of a spherical grain of radius $a$ with the velocity relative to gas $w_{\mathrm{d}}$ is given by (e.g., Draine \& Salpeter 1979)

$$
\frac{d w_{\mathrm{d}}}{d t}=-\frac{3 n_{\mathrm{H}} k T}{2 a \rho_{\mathrm{d}}} \sum_{i} A_{i} G_{i}\left(s_{i}\right)
$$

where $n_{\mathrm{H}}$ is the number density of hydrogen atom, $k$ is the Boltzmann constant, $T$ is the gas temperature, $\rho_{\mathrm{d}}$ is the bulk density of grain and $s_{i}^{2}=m_{i} w_{\mathrm{d}}^{2} / 2 k T$ with the mass $m_{i}$ of gas species $i$. The summation is taken over all gas species, and $A_{i}$ is the number abundance of gas species $i$ relative to that of hydrogen atom. For the function $G_{i}\left(s_{i}\right)$ whose exact formula is given by Baines, Williams, \& Asebiomo (1965), the following analytical approximation has been proposed by Draine \& Salpeter (1979)

$$
G_{i}\left(s_{i}\right) \approx \frac{8 s_{i}}{3 \sqrt{\pi}}\left(1+\frac{9 \pi}{64} s_{i}^{2}\right)^{\frac{1}{2}}
$$

with the accuracy within $1 \%$ for $0<s_{i}<\infty$. In the calculations, we adopt this formula. It can be seen from Equation (19), grains with smaller size and/or higher relative velocity can be effectively decelerated by drag force and come to comove with gas in shock.

\subsection{Grain destruction by sputtering}

The destruction of dust grains in high-velocity and high-temperature nonradiative shock is dominated by sputtering. Actually, we can neglect the thermal evaporation of dust, because the temperature of dust cannot be raised as high as their sublimation temperature ( 1000-2000 K) for the preshock gas density considered in this paper (e.g., Dwek 1987). Furthermore, we neglect the shattering process and partial vaporization caused by graingrain collisions, since the number density of dust particles is very small in postshock flow and collisions between dust grains are extremely rare without a magnetic field.

The erosion rate of grain by sputtering is calculated by taking the angle-averaged sputtering yield $\left\langle Y_{i}\left(E_{i}\right)\right\rangle_{\theta}=2 Y_{i}^{0}\left(E_{i}\right)$ (Draine \& Salpeter 1979) and is given by (e.g., Dwek et al. 1996)

$$
\frac{d a}{d t}=-\frac{m_{\mathrm{sp}}}{2 \rho_{\mathrm{d}}} n_{\mathrm{H}} \sum_{i} A_{i}\left(\frac{8 k T}{\pi m_{i}}\right)^{\frac{1}{2}} \frac{e^{-s_{i}^{2}}}{2 s_{i}} \int \sqrt{\epsilon_{i}} e^{-\epsilon_{i}} \sinh \left(2 s_{i} \sqrt{\epsilon_{i}}\right) Y_{i}^{0}\left(\epsilon_{i}\right) d \epsilon_{i}
$$


where $\epsilon_{i}=E_{i} / k T$ and $m_{\mathrm{sp}}$ is the average mass of the sputtered atoms. Equation (21) can be reduced to the following equations for the two extreme cases of $s_{i}$ (Dwek \& Arendt 1992; Tielens et al. 1994); one is for $s_{i} \ll 1$ where a stationary grain suffers the thermal sputtering caused by collisions originating from thermal motion of gas, and the erosion rate is written as

$$
\frac{1}{n_{\mathrm{H}}} \frac{d a}{d t}=-\frac{m_{\mathrm{sp}}}{2 \rho_{\mathrm{d}}} \sum_{i} A_{i}\left(\frac{8 k T}{\pi m_{i}}\right)^{\frac{1}{2}} \int \epsilon_{i} e^{-\epsilon_{i}} Y_{i}^{0}\left(\epsilon_{i}\right) d \epsilon_{i}
$$

The other is in the limit of $s_{i} \rightarrow \infty$ where the non-thermal sputtering erodes a hypersonic grain with the rate given by

$$
\frac{1}{n_{\mathrm{H}}} \frac{d a}{d t}=-\frac{m_{\mathrm{sp}}}{2 \rho_{\mathrm{d}}} w_{\mathrm{d}} \sum_{i} A_{i} Y_{i}^{0}\left(E=0.5 m_{i} w_{\mathrm{d}}^{2}\right) .
$$

Figure 2 shows the erosion rate of each dust species by sputtering in the gas with the primordial elemental composition of metallicity $Z=10^{-4} Z_{\odot}$ given in Table 1; Figure 2a for thermal sputtering versus the gas temperature $T$ from Equation (22) and Figure 2b for non-thermal sputtering versus the velocity of dust relative to gas $w_{\mathrm{d}}$ from Equation (23). The erosion rate by thermal (non-thermal) sputtering steeply increases from $T \sim 10^{5} \mathrm{~K}$ $\left(w_{\mathrm{d}} \sim 30 \mathrm{~km} \mathrm{~s}^{-1}\right)$, reaches a peak at $T=4-20 \times 10^{7} \mathrm{~K}\left(w_{\mathrm{d}}=500-1300 \mathrm{~km} \mathrm{~s}^{-1}\right)$, and then slowly decreases with increasing $T\left(w_{\mathrm{d}}\right)$. This behavior of the erosion rate as a function of $T$ and $w_{\mathrm{d}}$ reflects the dependence of sputtering yield on impact energy (see 5.2). Among dust species considered in this paper, $\mathrm{C}$ grain has the lowest erosion rate at $T \geq 2 \times 10^{6} \mathrm{~K}$ $\left(w_{\mathrm{d}} \geq 200 \mathrm{~km} \mathrm{~s}^{-1}\right)$, which is about one order of magnitude lower than that of FeS grain with the highest rate at $T \geq 10^{7} \mathrm{~K}\left(w_{\mathrm{d}} \geq 400 \mathrm{~km} \mathrm{~s}^{-1}\right)$. For thermal sputtering, the erosion rate $d a / d t$ for the other dust species is $\sim 1.2 \times 10^{-6} n_{\mathrm{H}} \mu \mathrm{m} \mathrm{yr} \mathrm{yr}^{-1} \mathrm{~cm}^{3}$ within a factor of three at $T \geq 2 \times 10^{6} \mathrm{~K}$. For non-thermal sputtering at $w_{\mathrm{d}} \leq 200 \mathrm{~km} \mathrm{~s}^{-1}$, the destruction by $\mathrm{He}^{+}$is dominant, and the erosion rate of $\mathrm{Al}_{2} \mathrm{O}_{3}$ is the lowest.

\subsection{Grain heating}

The collisions with gas can also heat dust grains in postshock flow. The collisional heating rate $H\left(a, T, n_{\mathrm{H}}\right)$ of dust grain with radius $a$ is presented by Dwek \& Arendt (1992) as

$$
H\left(a, T, n_{\mathrm{H}}\right)=n_{\mathrm{H}} \pi a^{2} k T \sum_{i} A_{i}\left(\frac{8 k T}{\pi m_{i}}\right)^{\frac{1}{2}} \frac{e^{-s_{i}^{2}}}{2 s_{i}} \int \epsilon_{i}^{\frac{3}{2}} e^{-\epsilon_{i}} \sinh \left(2 s_{i} \sqrt{\epsilon_{i}}\right) \eta_{i}\left(a, \epsilon_{i}\right) d \epsilon_{i}
$$


where $\eta_{i}\left(a, \epsilon_{i}\right)$ is the fraction of kinetic energy of gas species $i$ deposited into the dust grain. The values of $\eta_{i}\left(a, \epsilon_{i}\right)$ are calculated by comparing an effective grain thickness $a_{\text {eff }}(4 a / 3$ for a spherical grain) with the stopping range of the incident particle $l_{\mathrm{s}}$. When $a_{\mathrm{eff}} \geq$ $l_{\mathrm{s}}$, the incident particle deposits almost all of the energy into dust grains, otherwise, the difference between $a_{\text {eff }}$ and $l_{\mathrm{s}}$ is exploited to determine the fraction of deposited energy. The approximations for the stopping range of ion species considered here are given in Dwek (1987). For the electron stopping range, we adopt the approximation derived by Tabata, Ito, \& Okabe (1972) and Iskef, Cunningham, \& Watt (1983). This electron stopping range reduces the heating rate by $\sim 30 \%$ at $10^{6} \mathrm{~K} \lesssim T \lesssim 10^{9} \mathrm{~K}$ compared with that given by Dwek (1987). The values and detailed derivation of $\eta_{i}(a, E)$ will be presented elsewhere (Nozawa et al. in preparation).

\section{Results of calculation of dust destruction}

In this section, we show the results of numerical simulations for dust destruction in nonradiative shock, focusing on the results calculated for the ISM-dust specified by the unmixed grain model described in $\S 3$. In 7.1, we demonstrate the time evolution of temperature and density of gas and the destruction of dust grains in the interstellar shock that is driven by the SN model of $\mathrm{C} 20$ with $E_{51}=1$ and $M_{\mathrm{pr}}=20 M_{\odot}$ and is propagating into the ISM with

$n_{\mathrm{H}, 0}=1 \mathrm{~cm}^{-3}$. In 7.2 , we investigate the dependences of the destruction efficiency and the mass of gas swept up by shock on $E_{51}, M_{\mathrm{pr}}$ and $n_{\mathrm{H}, 0}$, and derive the approximation formula for the time-scale of destruction for each dust species in the early universe. The effects of the cooling processes of gas on the evolution of nonradiative shock and the destruction of dust are briefly discussed in 7.3 .

\subsection{Dust destruction in interstellar shock for C20 model}

\subsubsection{Time evolution of temperature and density of gas in shock}

Figure 3 shows the structures of density (upper panel) and temperature of gas (lower panel) in nonradiative shock at given times, and the solid lines in Figure 4 give the time evolution of the shock velocity $V_{\text {shock }}$ and the gas temperature $T_{\text {shock }}$ at the shock front. As shown in Figure 3, the gas density increases to 4 times that in the ISM and the gas temperature steeply raises up at the shock front which is indicated by the arrow for a given time step. With the initial shock velocity $\simeq 6000 \mathrm{~km} \mathrm{~s}^{-1}$, the gas temperature at the shock front remains above $10^{8} \mathrm{~K}$ until $t \simeq 200$ years, and both $V_{\text {shock }}$ and $T_{\text {shock }}$ decrease with time. 
At $t>5 \times 10^{4}$ years after the explosion, the density of gas in the inner postshock region is about one order of magnitude lower than that in the unshocked region, and the low-density hot bubble with temperature of several times $10^{6} \mathrm{~K}$ is formed (Fig. 3). In this model, $V_{\text {shock }}$ decelerates below $100 \mathrm{~km} \mathrm{~s}^{-1}$ at $t_{\mathrm{tr}} \simeq 10^{5}$ years when the shock front travels the distance of $\simeq 30 \mathrm{pc}$ and $T_{\text {shock }}$ drops down to several $10^{4} \mathrm{~K}$. After then, a dense thin-shell forms at the shock front because of the effective line cooling, and the SNR enters into radiative phase.

\subsubsection{Dynamics and destruction of dust grains in shock}

The motion of dust grains (upper panel) and the evolution of their sizes (lower panel) in postshock flow as a function of time are depicted in Figure 5, as an example for $\mathrm{C}$ grains with the initial size of $0.01 \mu \mathrm{m}$ (dashed), $0.1 \mu \mathrm{m}$ (dotted), and $1 \mu \mathrm{m}$ (solid). The thick solid curve in the upper panel indicates the position of the shock front. As dust grains initially at rest in the ISM intrude into the blast wave, they depict the different trajectories depending on their initial sizes, which clearly demonstrates that the dust grains are segregated and subjected to the different sputtering processes in the postshock flow; small grains with initial radius of $0.01 \mu \mathrm{m}$ are quickly decelerated by drag force, are trapped into gas near the shock front, and are completely destroyed by thermal sputtering. The $0.1 \mu \mathrm{m}$-sized grains are gradually decelerated to comove with gas in $7 \times 10^{3}$ years after entering into the shock. Large-sized grains with radius of $1 \mu \mathrm{m}$ are not so much suffered from deceleration by drag force and continue to keep the high velocity relative to gas. As a result, they are subjected to nonthermal sputtering, but their sizes are reduced very little, partly because they stay in the inner region of postshock flow where the gas density is lower than that near the shock front (see Fig. 3).

The modification of size distribution of each dust species due to destruction by sputtering is illustrated in Figure 6; Figure 6a for the initial size distribution before destruction and Figure $6 \mathrm{~b}$ for the size distribution after destruction. Since the erosion rate by sputtering does not strongly depend on grain size (see 6.2), small grains are predominantly destroyed regardless of grain species; the number of small-sized grain such as $\mathrm{Al}_{2} \mathrm{O}_{3}$ is greatly reduced. $\mathrm{C}, \mathrm{SiO}_{2}$ and $\mathrm{Fe}$ grains whose initial size distributions are lognormal with the relatively large average size are eroded but not completely destructed, and increase the numbers of smaller ones. Grains with the size larger than $0.1 \mu \mathrm{m}$ are little affected by erosion. Note that the size distribution summed up over all grains species gets flatter for the smaller radius with time compared with the corresponding initial size distribution approximated by a power-law formula with index of -2.5 , while that for the larger radius remains almost unchanged. 


\subsubsection{Efficiency of dust destruction}

The destruction efficiency $\epsilon_{j}$ of each grain species is given in Table 5 (see unmixed grain model) along with the initial average radius. It is expected that the efficiency of dust destruction are higher for grain species with the smaller average size because the smaller grains are predominantly destructed by sputtering. In fact, the efficiency of destruction of $\mathrm{Al}_{2} \mathrm{O}_{3}$ grain with the smallest average radius is 0.667 and is the highest among dust species considered here. Si and Fe grains with initial average radii larger than $0.1 \mu \mathrm{m}$ have the destruction efficiencies less than 0.2; 0.13 for $\mathrm{Si}$ grain and 0.15 for Fe grain. However, the destruction efficiency of $\mathrm{C}$ grain with the smaller average radius is smaller than that of $\mathrm{SiO}_{2}$ grain because of the lowest erosion rate among all grain species at $T \gtrsim 2 \times 10^{6} \mathrm{~K}$ (see Fig. 2). Also, FeS grain has higher destruction efficiency (0.578) than those of $\mathrm{Mg}_{2} \mathrm{SiO}_{4}(0.451)$ and $\mathrm{MgO}$ grains (0.505) despite of the larger initial average radius, which reflects not only the higher erosion rate at $T \gtrsim 10^{7} \mathrm{~K}$ but also the mass distribution much more weighted toward the smaller grains. Likewise, the destruction efficiency of $\mathrm{MgSiO}_{3}$ grain with average size comparable to that of $\mathrm{Mg}_{2} \mathrm{SiO}_{4}$ and $\mathrm{MgO}$ is high (0.637) because of the lack of large grains. These facts indicate that the efficiency of dust destruction depends on the initial size distribution of dust grains as well as on the sputtering yield.

In order to clarify the effect of the initial size distribution on the efficiency of dust destruction, we present the results of dust destruction for the mixed grain model calculated with the same SN model and value of $n_{\mathrm{H}, 0}$ as those for the unmixed grain model. Figure 7 shows the size distribution of each grain species $\left(\mathrm{Al}_{2} \mathrm{O}_{3}, \mathrm{MgSiO}_{3}, \mathrm{Mg}_{2} \mathrm{SiO}_{4}, \mathrm{SiO}_{2}\right.$ and $\left.\mathrm{Fe}_{3} \mathrm{O}_{4}\right)$ before destruction (Fig. 7a) and after destruction (Fig. 7b), and the destruction efficiencies of these grains are tabulated in Table 5 (see mixed grain model). As is the same as the unmixed grain model, the numbers of $\mathrm{Al}_{2} \mathrm{O}_{3}$ and $\mathrm{Fe}_{3} \mathrm{O}_{4}$ grains with the small average size ( $<$ a few tens $\AA$ ) are considerably reduced, which results in high efficiencies of destruction $(>0.7)$. For $\mathrm{MgSiO}_{3}, \mathrm{Mg}_{2} \mathrm{SiO}_{4}$ and $\mathrm{SiO}_{2}$ grains with lognormal size distributions, the erosion of large grains leads to the increase of the number of smaller ones, and their destruction efficiencies span the range of $0.4 \lesssim \epsilon_{j} \lesssim 0.64$. Note that although the average size is twice larger than that in the unmixed case, the destruction efficiency (0.59) of $\mathrm{Mg}_{2} \mathrm{SiO}_{4}$ grain in the mixed case is significantly larger than that (0.45) in the unmixed case. The reason is that $\mathrm{Mg}_{2} \mathrm{SiO}_{4}$ grains formed in the unmixed ejecta have power-law-like size distribution and include the large-sized grains of $>0.1 \mu \mathrm{m}$ almost undestructed in shock; the average size is not always suitable for assessing the feasibility of dust destruction. Thus, we conclude that the dust destruction efficiency is very sensitive to the initial size distribution. The mass fraction of dust destructed reaches up to $34 \%$ for the unmixed grain model and $50 \%$ for the mixed grain model. 


\subsection{Time-scale of dust destruction in the early universe}

In this subsection, we investigate the dependences of the efficiency of dust destruction $\epsilon_{j}$ and the mass of gas swept up by shock $M_{\text {swept }}$ on $E_{51}, M_{\mathrm{pr}}$ and $n_{\mathrm{H}, 0}$, and derive an analytic formula describing the time-scale of dust destruction for the unmixed grain model. Figure 8 shows the destruction efficiency of each grain species versus SN explosion energy calculated for $n_{\mathrm{H}, 0}=1 \mathrm{~cm}^{-3}$; Figure $8 \mathrm{a}$ is for $\mathrm{Al}_{2} \mathrm{O}_{3}, \mathrm{FeS}, \mathrm{Mg}_{2} \mathrm{SiO}_{4}$ and Fe grains, and Figure $8 \mathrm{~b}$ is for $\mathrm{MgSiO}_{3}, \mathrm{MgO}, \mathrm{SiO}_{2}, \mathrm{C}$ and $\mathrm{Si}$ grains. Also, the overall efficiency of dust destruction, which is defined as the ratio of the total mass of dust destructed to the total mass of dust swept up by shock, is plotted in Figure 8a. The SN models used for the calculations are distinguished by open circles (CCSNe), open squares (HNe) and filled triangles (PISNe).

The destruction efficiencies for each grain species are almost the same for CCSNe and a PISN with the explosion energy of $E_{51}=1$ irrespective of the progenitor mass $M_{\mathrm{pr}}$, and increase with increasing $E_{51}$. Note that high explosion energy with $E_{51} \geq 10$ causes the temperature of gas in postshock flow to raise up as high as $10^{9} \mathrm{~K}$, but this does not directly influence on the efficiency of dust destruction because the increase of gas temperature does not always lead to the enhancement of the erosion rate by sputtering (see Fig. 2a). The reason for the increased efficiency with increasing $E_{51}$ is considered as follows. The highvelocity shock (the initial shock velocity $\gtrsim 10^{4} \mathrm{~km} \mathrm{~s}^{-1}$ ) generated by the energetic SN explosion induces high velocity of dust relative to gas. Then, dust grains are efficiently decelerated by drag force (see Equation (19)), are trapped in the high-density region near the shock front, and are significantly eroded by thermal sputtering. Furthermore, since the high shock velocity takes much longer time to drop down to $100 \mathrm{~km} \mathrm{~s}^{-1}$ (see Fig. 9), dust grains are immersed in a hot plasma for a long time, which causes even larger grains to be more eroded.

It should be pointed out here that the efficiencies of destruction for the models of P170 with $E_{51}=20$ and P200 with $E_{51}=28$ are a little higher than that for H30 with $E_{51}=30$. The reason is considered that the ejecta mass of PISNe is more than 6 times larger than that of $\mathrm{HNe}$, and the resulting longer duration of the free expansion phase causes the longer truncation time than HNe (Fig. 9). On the other hand, the efficiency of destruction for P150 with $M_{\mathrm{pr}}=150 M_{\odot}$ and $E_{51}=1$ is almost the same as that for CCSNe with $E_{51}=1$ because the initial shock velocity $\left(\sim 3000 \mathrm{~km} \mathrm{~s}^{-1}\right)$ much lower than that of CCSNe makes the truncation time comparable to CCSNe. Therefore, although the destruction efficiency

$\epsilon_{j}$ is almost independent of $M_{\mathrm{pr}}$ as long as $E_{51}=1, \epsilon_{j}$ for PISNe higher than that for HNe at $E_{51} \geq 10$ reflects the difference in the explosion mechanism depending on the progenitor mass.

To examine the dependence of $\epsilon_{j}$ on $E_{51}$ for each type of SN, we calculate the coefficients 
$a_{1, j}$ and $b_{1, j}$ in Equation (A1) given in Appendix for SNe II (C13, C20, C25, C30, H25 and H30) and PISNe (P150, P170 and P200) separately, and the calculated overall efficiencies of dust destruction are indicated by dotted (SNe II) and dashed lines (PISNe) in Figure 8a. Although the difference in the efficiency of dust destruction between SNe II and PISNe increases with increasing $E_{51}$, the deviations from the values calculated by $a_{1, j}$ and $b_{1, j}$ for all SN models are at most about $10 \%$ at $E_{51}=30$. Thus, we consider that $\epsilon_{j}$ is almost independent of $M_{\mathrm{pr}}$ as long as $E_{51}$ is the same. For reference, we tabulate the values of $a_{1, j}$ and $b_{1, j}$ for SNe II and PISNe in Table 6. The mass of gas swept up by shock $M_{\text {swept }}$ and the truncation time $t_{\mathrm{tr}}$ calculated for $n_{\mathrm{H}, 0}=1 \mathrm{~cm}^{-3}$ are presented in Figure 9 as a function of SN explosion energy. As is the same as $\epsilon_{j}, M_{\text {swept }}$ and $t_{\mathrm{tr}}$ are almost the same for $E_{51}=1$ regardless of $M_{\mathrm{pr}}$, and increase with increasing $E_{51}$.

Next, we show the dependences of $\epsilon_{j}$ and $M_{\text {swept }}$ on the preshock gas density $n_{\mathrm{H}, 0}$. Figure 10a plots the overall efficiency of dust destruction versus $n_{\mathrm{H}, 0}$ for C20 (crosses), H25 (open circles) and H30 models (filled triangles) with $E_{51}=1,10$ and 30, respectively. The efficiency of dust destruction increases with increasing $n_{\mathrm{H}, 0}$, since higher gas density causes more frequently collisions between dust and gas to erode efficiently the surface of dust grains by sputtering; for example, the mass fraction of dust destroyed for H30 model is $78 \%$ for $n_{\mathrm{H}, 0}=10 \mathrm{~cm}^{-3}$, but only $23 \%$ for $n_{\mathrm{H}, 0}=0.1 \mathrm{~cm}^{-3}$. In Figure $10 \mathrm{~b}$, we present the mass of gas swept up by shock $M_{\text {swept }}$ as a function of $n_{\mathrm{H}, 0}$ for C20, H25 and H30 models. Note that $M_{\text {swept }}$ decreases with increasing $n_{\mathrm{H}, 0}$, because shock wave more quickly decelerates and travels only small distance.

The approximation formulae presented in Appendix being combined, the time-scale of destruction for each dust species by the interstellar shock in the early universe is presented by

$$
\tau_{\mathrm{SN}, j}^{-1}=\epsilon_{j}\left(E_{51}, n_{\mathrm{H}, 0}\right) \frac{4144 E_{51}^{0.8} n_{\mathrm{H}, 0}^{-0.142 E_{51}^{0.063}} M_{\odot}}{M_{\mathrm{ISM}}} \gamma_{\mathrm{SN}},
$$

for all SN models, where the dependences of $\epsilon_{j}$ on $E_{51}$ and $n_{\mathrm{H}, 0}$ are given by Equations (A1) and (A3), respectively. We derived the time-scale of dust destruction as a function of not only the explosion energy of SN but also the gas density in the ISM. The swept-up gas mass is proportional not to $E_{51}$ but to $\sim E_{51}^{0.8}$, being different from the formula proposed by McKee (1989). The time-scale of dust destruction derived here could be applicable to investigate the time evolution of dust grains in the early universe. Especially, the difference of the efficiency of dust destruction for each grain species may have great influences on the amount and size distribution of dust grains residing in the early interstellar space. 


\subsection{The effects of the cooling processes of gas on dust destruction}

Figure 11 shows the cumulative energy lost by the atomic process (dashed), the inverse Compton scattering (dotted) and the thermal emission from dust (solid) calculated for the SN model of $\mathrm{C} 20$ and for the ISM with parameters of $n_{\mathrm{H}, 0}=1$ and $Z=10^{-4} Z_{\odot}$ at the redshift of $z=20$. The total energy lost by these cooling processes is drawn by the thick solid curve. The inverse Compton cooling is comparable to that by the atomic process in the early phase of the SNR $\left(t<10^{4}\right.$ years). As the gas temperature decreases, the atomic process by $\mathrm{H}$ and $\mathrm{He}$ line coolings becomes dominant. Compared with the above two cooling processes, the thermal emission from dust is extremely low and contributes only less than 0.1 $\%$ to the total energy loss. The transition of nonradiative shock to radiative shock occurs when the cumulative energy loss reaches to $0.01 \%$ of the explosion energy. Thus, only the $\mathrm{H}$ and $\mathrm{He}$ line coolings affect the time evolution of nonradiative shock and the dust destruction efficiencies in the ISM with metallicity less than $Z=10^{-4} Z_{\odot}$ corresponding to the dust-to-gas mass ratio of $4.5 \times 10^{-7}$ in this paper.

However, it is expected that the effects of cooling of gas by dust come to be important for the ISM with higher dust-to-gas mass ratio. The result of the calculation for dust destruction in the ISM with $Z=10^{-4} Z_{\odot}$ and the dust-to-gas mass ratio $4.5 \times 10^{-3}$ shows that the truncation time and the overall efficiency of dust destruction decrease by $4.4 \%$ and $2.6 \%$, respectively, compared with the results for dust-to-gas mass ratio of $4.5 \times 10^{-7}$, though the gas temperature in postshock gas decreases by about $20 \%$. Even if the dust-to-gas mass ratio is raised up to by four orders of magnitude, the cooling of gas by dust in nonradiative shock does not have the significant effects on the efficiency of dust destruction as well as the evolution of nonradiative shock.

Furthermore, the inverse Compton cooling has influences on the evolution of nonradiative shock in the early universe. Because the cooling rate by the inverse Compton scattering is proportional to $n_{e} T(1+z)^{4}$ and that by atomic process is proportional to $n_{e} n_{\mathrm{H}} \sim n_{\mathrm{H}}^{2}$, the contribution of the inverse Compton cooling is enhanced in postshock flow with the lower gas density and/or the higher gas temperature at the higher redshift. At the redshift of $z=40$,

for the SN model C20 with the preshock gas density of $n_{\mathrm{H}, 0}=1 \mathrm{~cm}^{-3}$, the truncation time is only $2 \%$ shorter than that calculated at $z=20$. Thus, in this case, the inverse Compton cooling seems not to affect the destruction efficiency of dust grains. However, more systematic studies covering wide ranges of the SN explosion energy and the gas density in the ISM are necessary to reveal the effect of the inverse Compton cooling.

Note that the cooling function of gas for $Z=10^{-3} Z_{\odot}$ is almost the same as that for the zero metal case (Sutherland \& Dopita 1993). Thus, the parameters of the ISM considered in this paper $\left(Z=10^{-4} Z_{\odot}\right.$ and $\left.z=20\right)$ is competent for investigating dust destruction in 
the early universe, and the time-scale of dust destruction derived in 7.2 is applicable over the wide ranges of metallicity of gas in the $\operatorname{ISM}\left(Z \lesssim 10^{-3} Z_{\odot}\right)$ and redshift $(z \leq 40)$.

\section{Summary}

We investigate the destruction of dust grains in the interstellar shocks driven by $\mathrm{SNe}$ as the second step to reveal the evolution of dust in the early universe, based on the dust models obtained by Nozawa et al. (2003). We focus on dust destruction in nonradiative shock where dust grains are predominantly destroyed by non-thermal and thermal sputtering because of high temperature and high velocity of gas. The sputtering yields for the combinations of dust and ion species of interest to us are evaluated by applying the universal relation for sputtering yield with a slight modification and by determining the value of $K$ by fitting to the available experimental data and/or the results of the EDDY simulations. The modified universal relation derived here can present the better fits to the available experimental data than the previous one.

In the calculations of dust destruction, we solve the time evolution of gas temperature and density in spherically symmetric shocks, adopting the hydrodynamical models by Umeda \& Nomoto (2002) as the initial condition for interstellar shock and including the cooling of gas by the atomic process, the inverse Compton scattering and the thermal emission of dust. The erosion of dust by thermal and non-thermal sputtering caused by motion of dust relative to gas is carefully treated by taking into account the size distribution of each dust species. The results of calculations are summarized as follows.

(1) Because the sputtering predominantly destroys the small grain, the number of smallsized grain such as $\mathrm{Al}_{2} \mathrm{O}_{3}$ and $\mathrm{MgSiO}_{3}$ is greatly reduced. The erosion of $\mathrm{C}, \mathrm{SiO}_{2}$ and $\mathrm{Fe}$ grains whose size distributions are lognormal-like with the average size larger than $0.01 \mu \mathrm{m}$ increases the numbers of smaller ones. The size distribution summed up over all grains species becomes flatter for the small radius compared with the initial size distribution, while that for the radius larger than $0.2 \mu \mathrm{m}$ remains almost unchanged.

(2) The efficiency of dust destruction is higher for the grains with the small average size such as $\mathrm{Al}_{2} \mathrm{O}_{3}$ and $\mathrm{MgSiO}_{3}$ and with the power-law-like size distribution such as $\mathrm{FeS}, \mathrm{Mg}_{2} \mathrm{SiO}_{4}$ and $\mathrm{MgO}$. On the other hand, $\mathrm{Si}, \mathrm{Fe}, \mathrm{C}$ and $\mathrm{SiO}_{2}$ grains, which have the lognormal-like size distribution and the relatively large average radius, have lower efficiency of destruction. The detailed analysis of the behavior of dust destruction efficiency for each grain species indicates that not only sputtering yields but also the initial size distribution plays a crutial role in the efficiency of dust destruction by sputtering. 
(3) The efficiency of destruction $\epsilon_{j}$ for each dust species increases with increasing the explosion energy $E_{51}$ and/or with increasing the preshock gas density $n_{\mathrm{H}, 0}$, but is almost independent of the progenitor mass $M_{\mathrm{pr}}$ of SN as long as $E_{51}$ is the same. The destruction efficiency $\epsilon_{j}$ as a function of $E_{51}$ is well reproduced by a power-law formula given by Equation (A1). The dependence of $\epsilon_{j}$ on $n_{\mathrm{H}, 0}$ is well expressed by the quadratic equation (A3) in terms of $\log \left(n_{\mathrm{H}, 0}\right)$.

(4) As is the same as $\epsilon_{j}$, the mass of gas swept up by shock wave $M_{\text {swept }}$ is the increasing function of $E_{51}$ and is approximated by a power-law formula given by Equation (A2). However, $M_{\text {swept }}$ decreases with increasing the gas density in the ISM, and its dependence is also reproduced by a power-law formula whose index is given by Equation (A4) as a function of $E_{51}$. Finally, by combining these results, we present the analytic formula for the time-scale of destruction for each grain species in the early universe as a function of $E_{51}$ and $n_{\mathrm{H}, 0}$. The derived time-scale of dust destruction can be employed to investigate the time evolution of the amount of dust grains in the early universe.

(5) In the early universe, only the $\mathrm{H}$ and $\mathrm{He}$ line coolings affect the time evolution of nonradiative shock and the efficiencies of dust destruction. The thermal emission from dust grains is not so much important even for the ISM with the dust-to-gas mass ratio of $\sim 10^{-3}$ as long as the metallicity in the ISM is $Z \lesssim 10^{-3} Z_{\odot}$. Also, it is expected that the inverse Compton cooling does not have the great effects on the evolution of shock at high redshifts. Thus, the time-scale of dust destruction derived in this paper is applicable for ranges of metallicity of gas in the ISM of $Z \lesssim 10^{-3} Z_{\odot}$ and redshift of $z \leq 40$.

In this study, we focused on dust destruction in nonradiative shock where dust grains in the ISM are considered to be predominantly destroyed. We should be mentioned here that a part of dust grains formed in the ejecta of SNe are expected to be destroyed by reverse shock penetrating into the ejecta. Because sputtering is the most dominant destruction process of dust grains in reverse shock as well, the simulation code of dust destruction constructed in this paper can be applied to explore the destruction of dust grains by reverse shocks. This work is now in progress. Furthermore, in radiative shock with the shock velocities below 100 $\mathrm{km} \mathrm{s}^{-1}$, shattering and partial vaporization by grain-grain collisions become important in the presence of a magnetic field. A magnetic field frozen into the gas causes charged grains to gyrate and accelerate behind the shock front and results in the enhancement of not only the sputtering rate but also grain-grain collision frequency. The shattering by grain-grain collision mainly leads to the redistribution of grain size (Tielens et al. 1994; Jones, Tielens, \& Hollenbach 1996). The sophisticated study on this subject is left for the future work.

The authors are grateful to the anonymous referee for critical comments which have 
improved the manuscript. The authors thank Dr. H. Umeda and Prof. K. Nomoto for making the ejecta model of Pop III supernovae available. This work has been supported in part by a Grant-in-Aid for Scientific Research from the Japan Society for the Promotion of Sciences (13640229).

\section{A. The appriximation formulae for $\epsilon_{j}$ and $M_{\text {swept }}$}

In this Appendix, we present the approximate formulae for the efficiency of dust destruction $\epsilon_{j}$ and the mass of gas swept up by shock $M_{\text {swept }}$ as function of the SN explosion energy $E_{51}$ and the gas density $n_{\mathrm{H}, 0}$ in the ISM. The energy dependence of the destruction efficiency for each grain species can be well reproduced by a power-law formula given by

$$
\epsilon_{j}=a_{1, j} E_{51}^{b_{1, j}}
$$

The solid lines in Figure 8 indicate the least-squares fittings by this power-law formula for all SNe whose numerical coefficients $a_{1, j}$ and $b_{1, j}$ are tabulated in Table 6 . The dependence of $M_{\text {swept }}$ on $E_{51}$ is well fitted by a power-law formula

$$
M_{\text {swept }}=a_{2} E_{51}^{b_{2}}
$$

using the coefficients $a_{2}$ and $b_{2}$ given in Table 6, and is depicted in Figure 9 for all SNe (solid), SNe II (dotted), and PISNe (dashed). The destruction efficiency $\epsilon_{j}$ for each grains species $j$ as well as the overall destruction efficiency as a function of $n_{\mathrm{H}, 0}$ is expressed by

$$
\log \epsilon_{j}=c_{j}\left(\log n_{\mathrm{H}, 0}\right)^{2}+d_{j} \log n_{\mathrm{H}, 0}+e_{j}
$$

and the coefficients $c_{j}, d_{j}$ and $e_{j}$ derived by the least squares fittings are given in Table 7 for the models C20, H25 and H30, respectively, along with the coefficients for overall destruction efficiency. The solid curves in Figure 10a depict the overall destruction efficiencies calculated by Equation (A3) for the models C20, H25 and H30. This formula reproduces the destruction efficiencies calculated by the simulations with the accuracy less than $10 \%$ for $n_{\mathrm{H}, 0} \leq 10 \mathrm{~cm}^{-3}$. The calculated $M_{\text {swept }}$ can be reproduced by a power-law formula $M_{\text {swept }} \propto n_{\mathrm{H}, 0}^{g}$ where the index $g$ is weakly dependent on $E_{51}$ and is approximated by

$$
g=-0.142 E_{51}^{0.063}
$$

This formula gives good agreements with the results of simulations, as represented by the solid lines in Figure 10b. 


\section{REFERENCES}

Andersen, H. H., \& Bay, H. L. 1981, in Sputtering by Particle Bombardment I, ed. R. Behrisch (Berlin; Springer-Verlag), 145

Bach, H. 1970, Nucl. Instr. Meth., 84, 4

Bach, H., Kitzmann, I., \& Schroder, H. 1974, Radiation Effects, 21, 31

Baines, M. J., Williams, I. P., \& Asebiomo, A. S. 1965, MNRAS, 130, 63

Bertoldi, F., Carilli, C. L., Cox, P., Fan, X., Strauss, M. A., Beelen, A., Omont, A., \& Zylka, R. 2003, A\&A, 406, L55

Bianchi, S., \& Ferrara, A. 2005, MNRAS, 358, 379

Blank, P., \& Wittmaack, K. 1979, J. Appl. Phys., 50, 1519

Bohdansky, J. 1984, Nucl. Instr. Meth. Phys. Res. B2, 587

Bohdansky, J., Bay, H. L., \& Ottenberger, W. 1978, J. Nucl. Mat., 76-77, 163

Bohdansky, J., Bay, H. L., \& Roth, J. 1977, In Proc. 7th Int. Vacuum Congress and 3rd Int. Conf. on Solid Surfaces, ed. by Dobrozemsky, R., Rudenauer, F., Viehbock, F. P. \& Breth, A. (private publisher, Wien), P. 1509

Bohdansky, J., Roth, J., \& Sinha, M. K. 1976, In proc. 9th Symp. on Fusion Technology (Pergamon; London), p. 541

Bohdansky, J., Roth, J., \& Bay, H. L. 1980, J. Appl. Phys., 51, 2861

Bromm, V., \& Larson, R. B. 2004, ARA\&A, 42, 79

Cazaux, S., \& Spaans, M. 2004, ApJ, 611, 40

Chase, M. W., Davies, C. A., Downey, J. R., Frurip, D. J., McDonald, R. A., \& Syverud, A. N. 1985, JANAF Thermochemical Tables 3d ed. (J. Phys. Ch em. Ref. Data, 14, Suppl 1)

Coburn, J. W., Winters, H. F., \& Chuang, T. J. 1977, J. Appl. Phys., 48, 3532

Draine, B. T., \& Salpeter, E. E. 1979, ApJ, 231, 77

Dwek, E. 1987, ApJ, 322, 812 
Dwek, E. 1998, ApJ, 501, 643

Dwek, E., \& Arendt, R. G. 1992, ARA\&A, 30, 11

Dwek, E., Foster, S. M., \& Vancura, O. 1996, ApJ, 457, 244

Edwin, R. P. 1973, J. Phys. D, 6, 833

Eernisse, E. P. 1971, J. Appl. Phys., 42, 480

Field, D., May, P. W., Pineau des Forets, G., \& Flower, D. R. 1997, MNRAS, 285, 839

Gnedin, N. Y., Ferrara, A., \& Zweibel, E. G. 2000, ApJ, 539, 505

Hauser, M. G., \& Dwek, E. 2001, ARA\&A, 39, 249

Hechtl, E., Bohdansky, J., \& Roth, J. 1981, J. Nucl. Mat., 103-104, 333

Heger, A., \& Woosley, S. E. 2002, ApJ, 567, 532

Hirashita, H., \& Ferrara, A. 2002, MNRAS, 337, 921

Hirashita, H., Nozawa, T., Kozasa, T., Ishii, T. T., \& Takeuchi, T. T. 2005, 357, 1077

Hirooka, Y. 2001, http://dppwi.nifs.ac.jp/

Ikeuchi, S., \& Ostriker, J. P. 1986, ApJ, 301, 522

Iskef, H., Cunningham, J. W., \& Watt, D. E. 1983, Phys. Med. Biol., 28, 535

Jones, A. P., Tielens, A. G. G. M., Hollenbach, D. J., \& McKee, C. F. 1994, ApJ, 433, 797

Jones, A. P., Tielens, A. G. G. M., \& Hollenbach, D. J. 1996, ApJ, 469, 740

Kitayama, T., Yoshida, N., Susa, H., \& Umemura, M. 2004, ApJ, 613, 631

Kozasa, T., Hasegawa, H., \& Nomoto, K. 1989, ApJ, 344, 325

Laegreid, N., \& Wehner, G. K. 1961, J. Appl. Phys., 32, 365

Loeb, A., \& Haiman, Z. 1997, ApJ, 490, 571

Machida, M. N., Tomisaka, K., Nakamura, F., \& Fujimoto, M. Y. 2005, ApJ, 622, 39

Maiolino, R., Oliva, E., Ghinassi, F., Pedani, M., Mannucci, F., Mujica, R., \& Juarez, Y. 2004a, A\&A, 420, 889 
Maiolino, R., Schneider, R., Oliva, E., Bianchi, S., Ferrara, A., Mannucci, F., Pedani, M., \& Roca Sogorb, M. 2004b, Nature, 431, 533

Mair, G., Müllar, E., Hillebrandt, W., \& Arnold, C. N. 1988, A\&A, 199, 114

Matsunami, N., Yamamura, Y., Itakawa, W., Itoh, N., Kazumata, Y., Myagawa, S., Morita, K., \& Shimizu, R. 1980, Radiation Effects Lett., 57, 15

May, P. W., Pineau des Forets, G., Flower, D. R., Field, D., Allan, N. L., \& Purton, J. A. 2000, MNRAS, 318, 809

McKee, C. F. 1989, in Interstellar Dust, ed. L. J. Allamandora \& A. G. G. M. Tielens (Dordrechent: Kluwer), 431

McKee, C. F., Hollenbach, D. J., Seab, C. G., \& Tielens, A. G. G. M. 1987, ApJ, 318, 674

Nenadovic, T., Perraillon, B., Bogdanov, Z., Djordjevic, Z., \& Milic, M. 1990, Nucl. Instr. Meth. Phys. Res., B48, 538

Nozawa, T., Kozasa, T., Umeda, H., Maeda, K., \& Nomoto, K. 2003, ApJ, 598, 785

Omukai, K., Tsuribe, T., Schneider, R., \& Ferrara, A. 2005, ApJ, 626, 627

Priddey, R. S., Isaak, K. G., McMahon, R. G., Robson, E. I., \& Pearson, C. P. 2003, MNRAS, 344, L74

Robson, I., Priddey, R. S., Isaak, K. G., \& McMahon, R. G. 2004, MNRAS, 351, L29

Rosenberg, D., \& Wehner, G. K. 1962, J. Appl. Phys., 33, 1842

Roth, J. 1983, in Sputtering by Particle Bombardment II, ed. R. Behrisch (Berlin; SpringerVerlag), 91

Roth, J., Bohdansky, J., \& Ottenberger, W. 1979, Rpt. IPP. 9/26 (MPI Plasmaphysik, Garching)

Schneider, R., Ferrara, A., Natarajan, P., \& Omukai, K. 2002, ApJ, 571, 30

Schneider, R., Ferrara, A., Salvaterra, R., Omukai, K., \& Bromm, V. 2003, Nature, 422, 869

Schneider, R., Ferrara, A., \& Salvaterra, R. 2004, MNRAS, 351, 1379

Sigmund, P. 1969, Phys. Rev., 184, 383 
Sigmund, P. 1981, in Sputtering by Particle Bombardment I, ed. R. Behrisch (Berlin; Springer-Verlag), 9

Sommerfeldt, H., Mashkova, E. S., \& Molchanov, V. A. 1972, Phys. lett., 38A, 237

Southern, A. L., Willis, W. R., \& Robinson, M. T. 1963, J. Appl. Phys., 34, 153

Sutherland, R. S., \& Dopita, M. A. 1993, ApJS, 88, 253

Tabata, T., Ito, R., \& Okabe, S. 1972, Nucl. Instr. Meth., 103, 85

Takeuchi, T. T., Hirashita, H., Ishii, T. T., Hunt, L. K., \& Ferrara, A. 2003, MNRAS, 343, 839

Takeuchi, T. T., Ishii, T. T., Nozawa, T., Kozasa, T., \& Hirashita, H. 2005, MNRAS, 362, 592

Tan, J. C., \& McKee, C. F. 2004, ApJ, 603, 383

Tielens, A. G. G. M., McKee, C. F., Seab, C. G., \& Hollenbach, D. J. 1994, ApJ, 431, 321

Todini, P., \& Ferrara, A. 2001, MNRAS, 325, 726

Umeda, H., \& Nomoto, K. 2002, ApJ, 565, 385

van Albada, G. D., van Leer, B., \& Roberts, W. W. Jr. 1982, A\&A, 108, 76

Vanzi, L., Hunt, L. K., Thuan, T. X., \& Izotov, Y. I. A\&A, 363, 493

von Seefeld, H., Schmidl, H., Behrisch, R., \& Scherzer, B. M. U. 1976, J. Nucl. Mat., 63, 215

Wolfire, M. G., Hollenbach, D., McKee, C. F., Tielens, A. G. G. M., \& Bakes, E. L. O. 1995, ApJ, 443, 152

Ziegler, J. F., Biersack, J. P., \& Littmark, U. 1985, The stopping and Range of Ions in Solids. Pergamon, New York 
Table 1. Elemental Abundances of Gas in the ISM

\begin{tabular}{cccccc}
\hline \hline$Z / Z_{\odot}$ & $A_{\mathrm{He}}$ & $A_{\mathrm{C}}$ & $A_{\mathrm{N}}$ & $A_{\mathrm{O}}$ & $A_{\mathrm{e}}$ \\
\hline $10^{-4}$ & $8.04 \times 10^{-2}$ & $3.62 \times 10^{-8}$ & $1.12 \times 10^{-8}$ & $2.70 \times 10^{-7}$ & 1.128 \\
\hline
\end{tabular}

Note. - The number abundance $A_{i}$ relative to that of hydrogen atom in the ISM. Note that the abundances of $\mathrm{C}, \mathrm{N}$ and $\mathrm{O}$ are calculated by multiplying the metallicity of $Z=10^{-4} Z_{\odot}$ and the primordial ratios in Table 4 of Sutherland \& Dopita (1993). 
Table 2. Dust Models Used in the Calculations

\begin{tabular}{lccccc}
\hline \hline & \multicolumn{5}{c}{ unmixed grain model } \\
dust species & $\rho_{\mathrm{d}, j}\left(\mathrm{~g} \mathrm{~cm}^{-3}\right)$ & $A_{j}$ & $a_{\text {ave }, j}(\mu \mathrm{m})$ & $A_{j}$ & $a_{\text {ave }, j}(\mu \mathrm{m})$ \\
\hline $\mathrm{C}$ & 2.26 & 0.103 & 0.029 & & \\
$\mathrm{Si}$ & 2.32 & 0.304 & 0.250 & & \\
$\mathrm{Fe}$ & 7.89 & 0.050 & 0.148 & & \\
$\mathrm{FeS}$ & 4.84 & 0.156 & 0.0088 & & \\
$\mathrm{Al}_{2} \mathrm{O}_{3}$ & 3.98 & 0.002 & 0.0007 & 0.001 & 0.0003 \\
$\mathrm{MgSiO}_{3}$ & 3.18 & 0.010 & 0.0045 & 0.102 & 0.011 \\
$\mathrm{Mg}_{2} \mathrm{SiO}_{4}$ & 3.20 & 0.219 & 0.0066 & 0.205 & 0.010 \\
$\mathrm{SiO}_{2}$ & 2.64 & 0.100 & 0.047 & 0.588 & 0.033 \\
$\mathrm{MgO}_{\mathrm{Fe}_{3} \mathrm{O}_{4}}^{3.56}$ & 0.056 & 0.0058 & & \\
\hline & 5.21 & & & 0.103 & 0.0022 \\
\hline
\end{tabular}

Note. - The models of dust grains are adopted from the results of the calculations of dust formation in the ejecta of Pop III SNe with $M_{\mathrm{pr}}=20$ $M_{\odot}$ by Nozawa et al. (2003). The bulk density of grain species $j$ is denoted by $\rho_{\mathrm{d}, j}$. The mass fraction $A_{j}$ and the average size $a_{\mathrm{ave}, j}$ of each grain species are given for the unmixed and mixed grain models. Note that the average radius of $\mathrm{Si}$ grain in the unmixed grain model is calculated for the grains larger than $0.03 \mu \mathrm{m}$ which occupy $99.8 \%$ of the total mass. 
Table 3. Models of Supernova Ejecta

\begin{tabular}{lcc}
\hline \hline Model & $\begin{array}{c}\text { Progenitor mass } \\
\left(M_{\odot}\right)\end{array}$ & $\begin{array}{c}\text { Explosion energy } \\
E_{51}\left(10^{51} \text { ergs }\right)\end{array}$ \\
\hline C13 & 13 & 1 \\
C20 & 20 & 1 \\
C25 & 25 & 1 \\
C30 & 30 & 1 \\
H25 & 25 & 10 \\
H30 & 30 & 30 \\
P150 & 150 & 1 \\
P170 & 170 & 20 \\
P200 & 200 & 28 \\
\hline
\end{tabular}

Note. - The models of the SN ejecta are adopted from Umeda \& Nomoto (2002). In the model names, the labels $\mathrm{C}, \mathrm{H}$ and $\mathrm{P}$ represent CCSNe, HNe and PISNe, respectively, and the numerical value denotes the mass of the progenitor in units of solar mass. 
Table 4. Parameters for Calculations of Sputtering Yield and References for Yield Data

\begin{tabular}{lccccl}
\hline \hline dust species & $U_{0}(\mathrm{eV})$ & $Z_{\mathrm{d}}$ & $M_{\mathrm{d}}$ & $K$ & \multicolumn{1}{c}{ references } \\
\hline $\mathrm{C}$ & 4.0 & 6 & 12 & 0.61 & $1,2,3,4,5$ \\
$\mathrm{Si}$ & 4.66 & 14 & 28 & 0.43 & $3,4,6,7,8,9,10,11$ \\
$\mathrm{Fe}$ & 4.31 & 26 & 56 & 0.23 & $3,4,5,7,8,12,13$ \\
$\mathrm{FeS}$ & 4.12 & 21 & 44 & 0.18 & EDDY \\
$\mathrm{Al}_{2} \mathrm{O}_{3}$ & 8.5 & 10 & 20.4 & 0.08 & $3,14,15$ \\
$\mathrm{MgSiO}_{3}$ & 6.0 & 10 & 20 & 0.1 & 16 \\
$\mathrm{Mg}_{2} \mathrm{SiO}_{4}$ & 5.8 & 10 & 20 & 0.1 & 16 \\
$\mathrm{SiO}_{2}$ & 6.42 & 10 & 20 & 0.1 & $3,14,15,17,18$ \\
$\mathrm{MgO}$ & 5.17 & 10 & 20 & 0.06 & 14,15, EDDY \\
$\mathrm{Fe}_{3} \mathrm{O}_{4}$ & 4.98 & 15.7 & 33.1 & 0.15 & EDDY \\
\hline
\end{tabular}

Note. - The surface binding energy is represented by $U_{0}$ in units of $\mathrm{eV}$. The mean atomic number and mean mass number of the target material are $Z_{\mathrm{d}}$ and $M_{\mathrm{d}}$, respectively, and the free parameter $K$ is determined by fitting to the available experimental data and/or the results of the EDDY simulation. See text for details.

References. - (1) Bohdansky, Bay, \& Ottenberger (1978); (2) Bohdansky, Roth, \& Sinha (1976); (3) Roth, Bohdansky, \& Ottenberger (1979); (4) Rosenberg \& Wehner (1962); (5) Hechtl, Bohdansky, \& Roth (1981); (6) Laegreid \& Wehner (1961); (7) Southern, Willis, \& Robinson (1963); (8) Eernisse (1971); (9) Sommerfeldt, Mashkova, \& Molchanov (1972); (10) Coburn, Winters, \& Chuang (1977); (11) Blank \& Wittmaack (1979); (12) von Seefeld et al. (1976); (13) Bohdansky, Bay, \& Roth (1977); (14) Bach (1970); (15) Nenadovic et al. (1990); (16) Tielens et al. (1994); (17) Bach, Kitzmann, \& Schroder (1974); (18) Edwin (1973) 
Table 5. The Efficiency of Dust Destruction for C20 Model with $n_{\mathrm{H}, 0}=1$

\begin{tabular}{lccccc}
\hline \hline & \multicolumn{3}{c}{ unmixed grain model } & \multicolumn{3}{c}{ mixed grain model } \\
& $\epsilon_{j}$ & $a_{\text {ave }, j} \quad(\mu \mathrm{m})$ & $\epsilon_{j}$ & $a_{\text {ave }, j} \quad(\mu \mathrm{m})$ \\
\hline $\mathrm{C}$ & 0.247 & 0.029 & & \\
$\mathrm{Si}$ & 0.134 & 0.250 & & \\
$\mathrm{Fe}$ & 0.154 & 0.148 & & \\
$\mathrm{FeS}$ & 0.578 & 0.0088 & & \\
$\mathrm{Al}_{2} \mathrm{O}_{3}$ & 0.667 & 0.0007 & 0.794 & 0.0003 \\
$\mathrm{MgSiO}_{3}$ & 0.637 & 0.0045 & 0.631 & 0.011 \\
$\mathrm{Mg}_{2} \mathrm{SiO}_{4}$ & 0.451 & 0.0066 & 0.586 & 0.010 \\
$\mathrm{SiO}_{2}$ & 0.411 & 0.047 & 0.399 & 0.033 \\
$\mathrm{MgO}$ & 0.505 & 0.0058 & & \\
$\mathrm{Fe}_{3} \mathrm{O}_{4}$ & \multicolumn{4}{|c}{0.741} & 0.0022 \\
overall & 0.340 & & 0.497 & \\
\hline
\end{tabular}


Table 6. Coefficients for the Approximation Formula (A1) and (A2)

\begin{tabular}{|c|c|c|c|c|c|c|}
\hline \multirow[b]{2}{*}{ dust species } & \multicolumn{2}{|c|}{ for all $\mathrm{SNe}$} & \multicolumn{2}{|c|}{ for SNe II } & \multicolumn{2}{|c|}{ for PISNe } \\
\hline & $a_{1, j}$ & $b_{1, j}$ & $a_{1, j}$ & $b_{1, j}$ & $a_{1, j}$ & $b_{1, j}$ \\
\hline $\mathrm{C}$ & $2.532 \mathrm{E}-01$ & $1.660 \mathrm{E}-01$ & $2.526 \mathrm{E}-01$ & $1.407 \mathrm{E}-01$ & $2.580 \mathrm{E}-01$ & $1.820 \mathrm{E}-01$ \\
\hline $\mathrm{Si}$ & $1.380 \mathrm{E}-01$ & $2.371 \mathrm{E}-01$ & $1.377 \mathrm{E}-01$ & $1.979 \mathrm{E}-01$ & $1.414 \mathrm{E}-01$ & $2.631 \mathrm{E}-01$ \\
\hline $\mathrm{Fe}$ & $1.583 \mathrm{E}-01$ & $2.402 \mathrm{E}-01$ & $1.586 \mathrm{E}-01$ & $2.005 \mathrm{E}-01$ & $1.594 \mathrm{E}-01$ & $2.707 \mathrm{E}-01$ \\
\hline $\mathrm{FeS}$ & $5.842 \mathrm{E}-01$ & 7.897E-02 & $5.850 \mathrm{E}-01$ & $6.758 \mathrm{E}-02$ & $5.834 \mathrm{E}-01$ & $8.857 \mathrm{E}-02$ \\
\hline $\mathrm{Al}_{2} \mathrm{O}_{3}$ & $6.724 \mathrm{E}-01$ & $5.649 \mathrm{E}-02$ & $6.733 \mathrm{E}-01$ & 4.803E-02 & $6.709 \mathrm{E}-01$ & 6.393E-02 \\
\hline $\mathrm{MgSiO}_{3}$ & $6.429 \mathrm{E}-01$ & $6.358 \mathrm{E}-02$ & $6.438 \mathrm{E}-01$ & $5.421 \mathrm{E}-02$ & $6.417 \mathrm{E}-01$ & $7.165 \mathrm{E}-02$ \\
\hline $\mathrm{Mg}_{2} \mathrm{SiO}_{4}$ & $4.585 \mathrm{E}-01$ & $1.146 \mathrm{E}-01$ & $4.590 \mathrm{E}-01$ & $9.827 \mathrm{E}-02$ & $4.595 \mathrm{E}-01$ & $1.275 \mathrm{E}-01$ \\
\hline $\mathrm{SiO}_{2}$ & $4.183 \mathrm{E}-01$ & $1.238 \mathrm{E}-01$ & $4.189 \mathrm{E}-01$ & $1.054 \mathrm{E}-01$ & 4.19E-01 & $1.383 \mathrm{E}-01$ \\
\hline $\mathrm{MgO}$ & $5.117 \mathrm{E}-01$ & $1.046 \mathrm{E}-01$ & $5.122 \mathrm{E}-01$ & $9.020 \mathrm{E}-02$ & $5.124 \mathrm{E}-01$ & $1.160 \mathrm{E}-01$ \\
\hline overall & $3.458 \mathrm{E}-01$ & $1.307 \mathrm{E}-01$ & $3.460 \mathrm{E}-01$ & $1.099 \mathrm{E}-01$ & $3.476 \mathrm{E}-01$ & $1.463 \mathrm{E}-01$ \\
\hline & $a_{2}$ & $b_{2}$ & $a_{2}$ & $b_{2}$ & $a_{2}$ & $b_{2}$ \\
\hline$M_{\text {swept }}$ & $4.144 \mathrm{E}+03$ & $7.967 \mathrm{E}-01$ & $4.118 \mathrm{E}+03$ & $6.627 \mathrm{E}-01$ & $4.481 \mathrm{E}+03$ & $8.864 \mathrm{E}-01$ \\
\hline
\end{tabular}


Table 7. Coefficients for the Approximation (A3)

\begin{tabular}{lccccccccc}
\hline \hline & \multicolumn{3}{c}{$\mathrm{C} 20$} & \multicolumn{4}{c}{$\mathrm{H} 25$} \\
dust species & $c_{j}$ & $d_{j}$ & $e_{j}$ & $c_{j}$ & $d_{j}$ & $e_{j}$ & $c_{j}$ & $d_{j}$ & $e_{j}$ \\
\hline $\mathrm{C}$ & -0.062 & 0.41 & -0.61 & -0.07 & 0.36 & -0.46 & -0.08 & 0.33 & -0.39 \\
$\mathrm{Si}$ & -0.044 & 0.54 & -0.87 & -0.06 & 0.50 & -0.67 & -0.072 & 0.47 & -0.58 \\
$\mathrm{Fe}$ & -0.057 & 0.55 & -0.81 & -0.078 & 0.49 & -0.61 & -0.096 & 0.46 & -0.51 \\
$\mathrm{FeS}$ & -0.088 & 0.23 & -0.23 & -0.077 & 0.18 & -0.16 & -0.073 & 0.16 & -0.13 \\
$\mathrm{Al}_{2} \mathrm{O}_{3}$ & -0.031 & 0.14 & -0.17 & -0.030 & 0.12 & -0.13 & -0.032 & 0.11 & -0.11 \\
$\mathrm{MgSiO}_{3}$ & -0.060 & 0.18 & -0.19 & -0.057 & 0.14 & -0.13 & -0.055 & 0.12 & -0.11 \\
$\mathrm{Mg}_{2} \mathrm{SiO}_{4}$ & -0.087 & 0.31 & -0.35 & -0.092 & 0.25 & -0.24 & -0.097 & 0.22 & -0.19 \\
$\mathrm{SiO}_{2}$ & -0.086 & 0.33 & -0.39 & -0.093 & 0.27 & -0.27 & -0.097 & 0.24 & -0.22 \\
$\mathrm{MgO}_{\text {overall }}$ & -0.079 & 0.29 & -0.30 & -0.086 & 0.23 & -0.20 & -0.089 & 0.20 & -0.16 \\
\hline & -0.067 & 0.33 & -0.47 & -0.067 & 0.29 & -0.35 & -0.072 & 0.26 & -0.30 \\
\hline
\end{tabular}



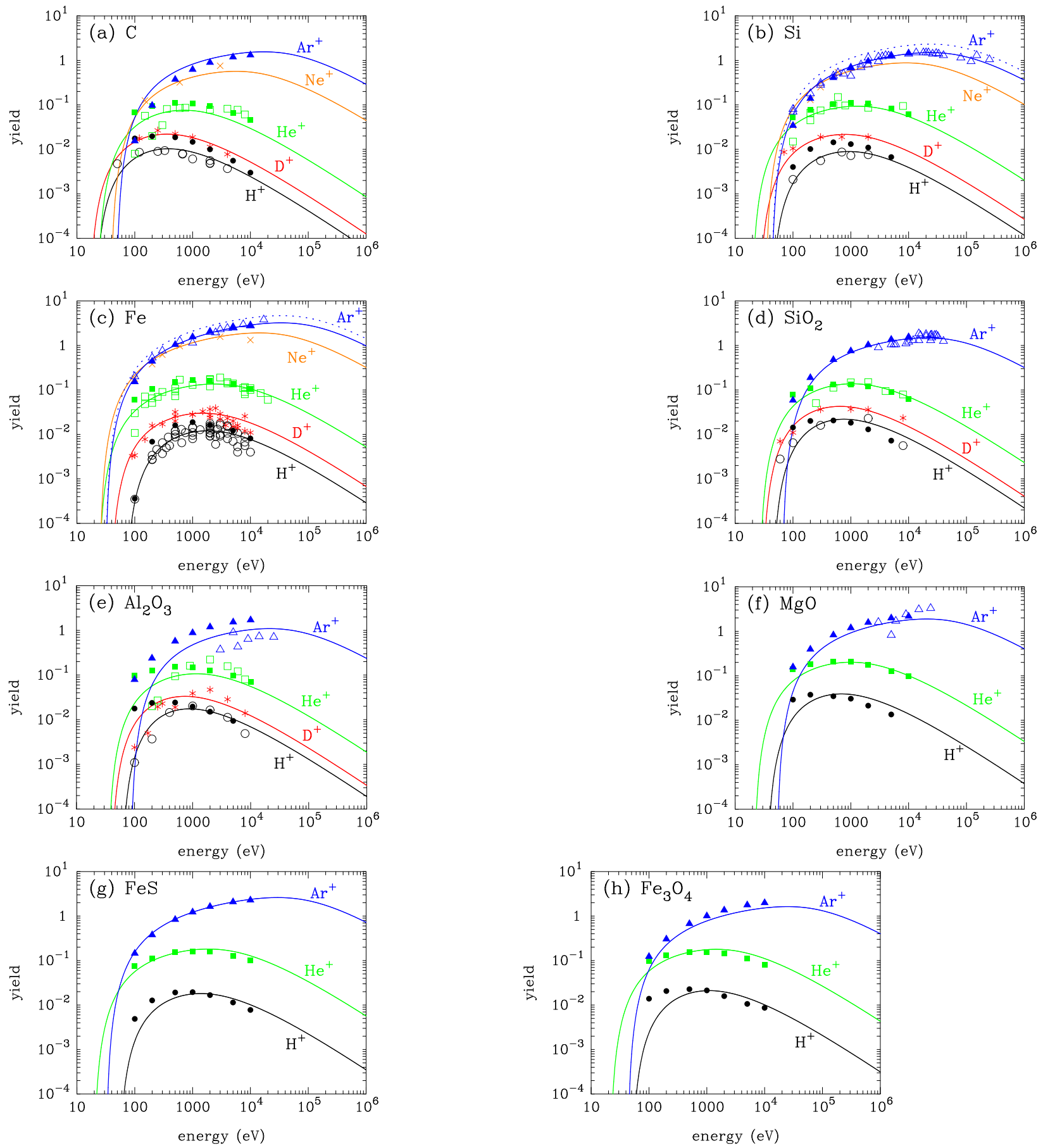

Fig. 1. - Sputtering yields of each grain species versus incident energy of projectiles. (a) C, (b) $\mathrm{Si}$, (c) $\mathrm{Fe}$, (d) $\mathrm{SiO}_{2}$, (e) $\mathrm{Al}_{2} \mathrm{O}_{3}$, (f) $\mathrm{MgO}$, (g) FeS, and (h) $\mathrm{Fe}_{3} \mathrm{O}_{4}$. The incident ion species are $\mathrm{H}^{+}$, $\mathrm{D}^{+}, \mathrm{He}^{+}, \mathrm{Ne}^{+}$and $\mathrm{Ar}^{+}$. The experimental data on sputtering yield are represented by open circles $\left(\mathrm{H}^{+}\right)$, asterisks $\left(\mathrm{D}^{+}\right)$, open squares $\left(\mathrm{He}^{+}\right)$, crosses $\left(\mathrm{Ne}^{+}\right)$and open triangles $\left(\mathrm{Ar}^{+}\right)$, and the results of sputtering yield calculated by the EDDY code are denoted by filled circles $\left(\mathrm{H}^{+}\right)$, filled squares $\left(\mathrm{He}^{+}\right)$and filled triangles $\left(\mathrm{Ar}^{+}\right)$. The solid curves show the best-fitting theoretical yields calculated by the universal relation. In (b) and (c), the dotted curves show the theoretical sputtering yields by $\mathrm{Ar}^{+}$projectile calculated by adopting $\alpha_{i}$ given by Equation (17). See the electronic edition of the Journal for a color version of this figure. 

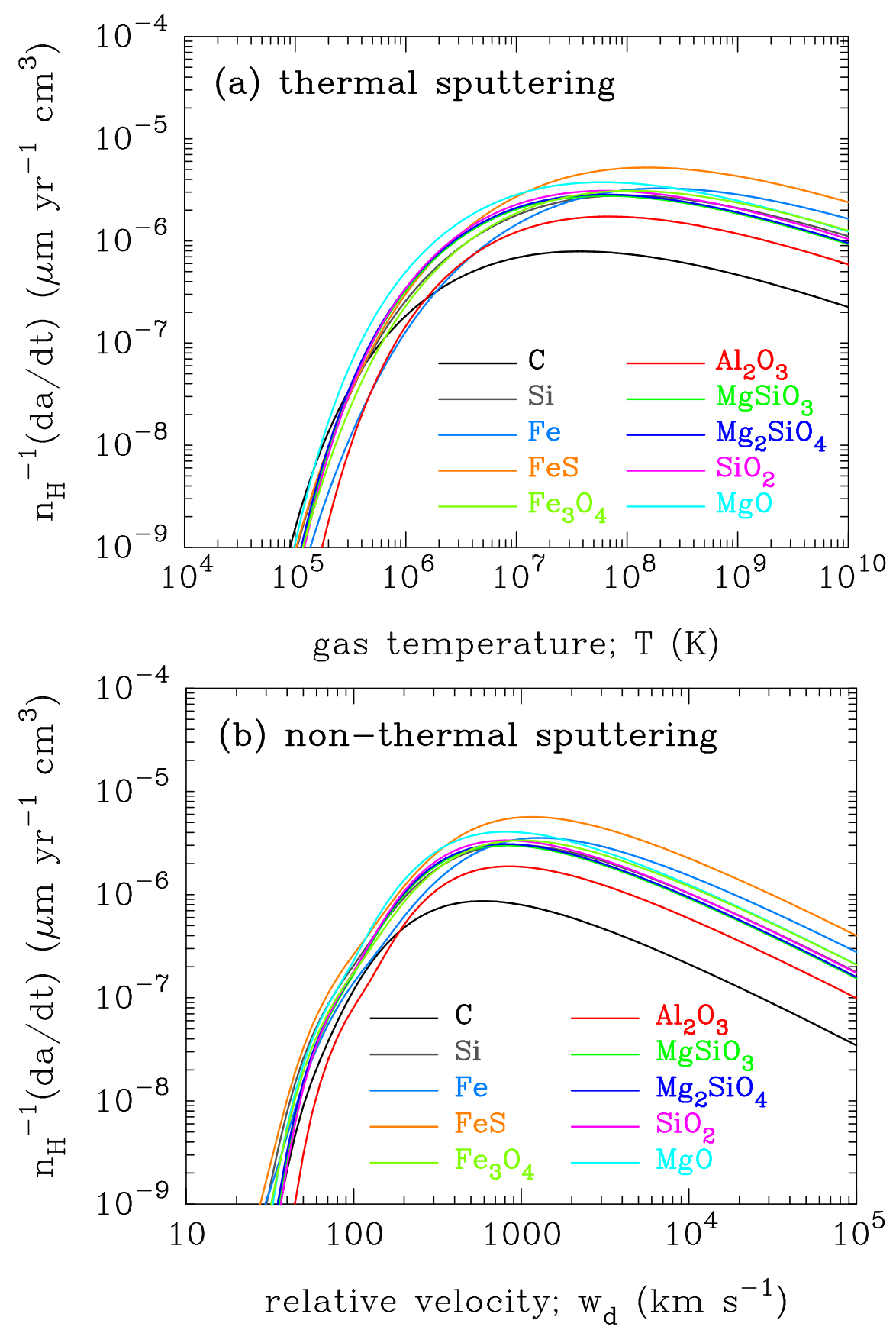

Fig. 2.- The erosion rate of each dust species by sputtering in units of $\mu \mathrm{m} \mathrm{yr}^{-1} \mathrm{~cm}^{3}$ calculated for the elemental composition of gas with $Z=10^{-4} Z_{\odot}$ given in Table 1 ; (a) the thermal sputtering calculated by Equation (22) as a function of gas temperature $T$ and (b) the non-thermal sputtering calculated by Equation (23) as a function of velocity of dust relative to gas $w_{\mathrm{d}}$. See the electronic edition of the Journal for a color version of this figure. 


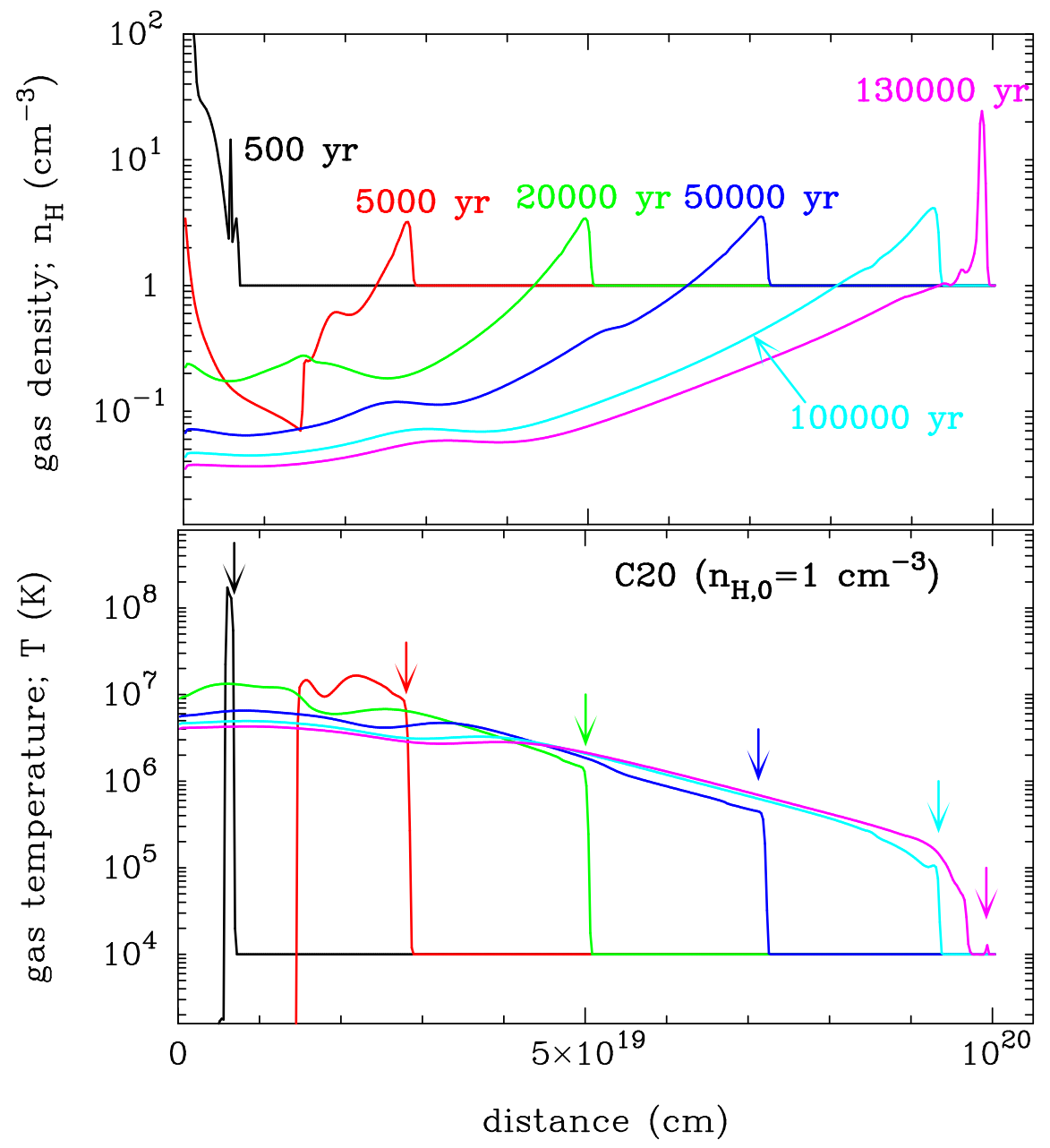

Fig. 3.- The structures of density (upper panel) and temperature of gas (lower panel) at given times in the interstellar shock that is driven by the SN model of C20 with $E_{51}=1$ and $M_{\mathrm{pr}}=20 M_{\odot}$ and is propagating into the ISM with $n_{\mathrm{H}, 0}=1 \mathrm{~cm}^{-3}$. The arrow in the lower panel indicates the position of the shock front at a given time. See the electronic edition of the Journal for a color version of this figure. 


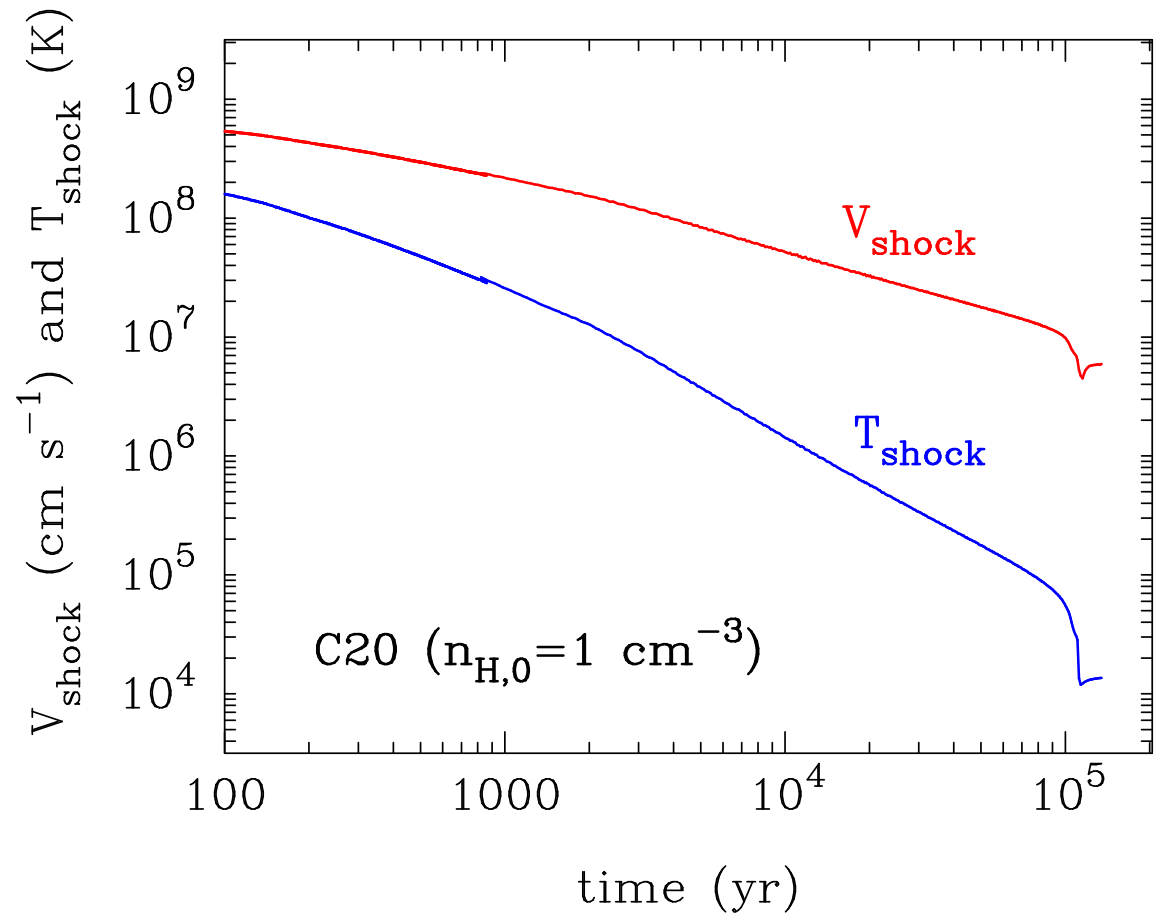

Fig. 4. - The time evolution of the shock velocity $V_{\text {shock }}$ and the gas temperature $T_{\text {shock }}$ at the shock front for model C20 with $n_{\mathrm{H}, 0}=1 \mathrm{~cm}^{-3}$. See the electronic edition of the Journal for a color version of this figure. 


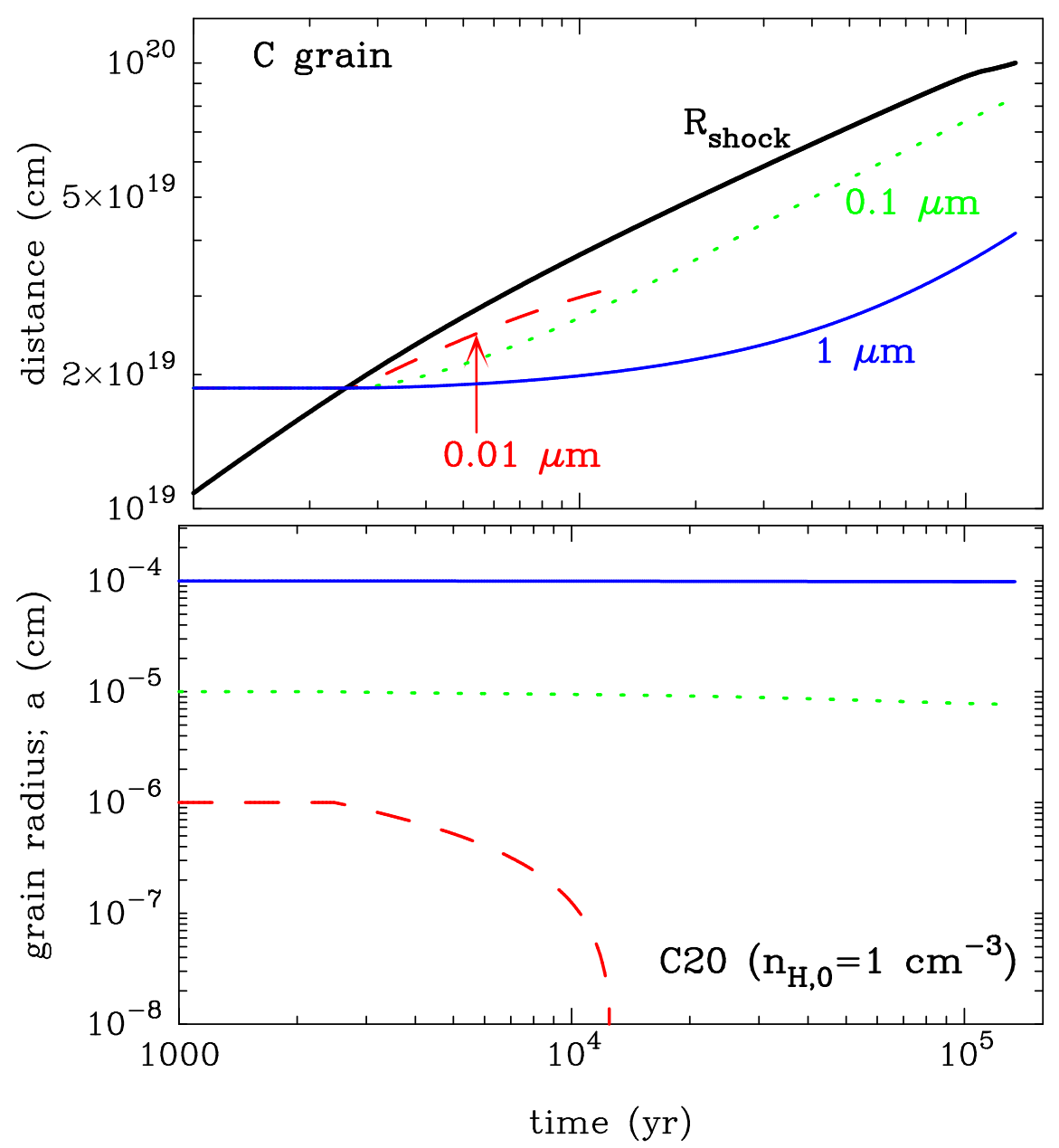

Fig. 5.- The time evolution of position (upper panel) and size (lower panel) of dust grain in postshock flow for model C20 with $n_{\mathrm{H}, 0}=1 \mathrm{~cm}^{-3}$. Dust species considered is C grain with the size of $0.01 \mu \mathrm{m}$ (dashed), $0.1 \mu \mathrm{m}$ (dotted) and $1 \mu \mathrm{m}$ (solid). The thick solid curve in the upper panel denotes the position of the shock front $R_{\text {shock }}$. See the electronic edition of the Journal for a color version of this figure. 

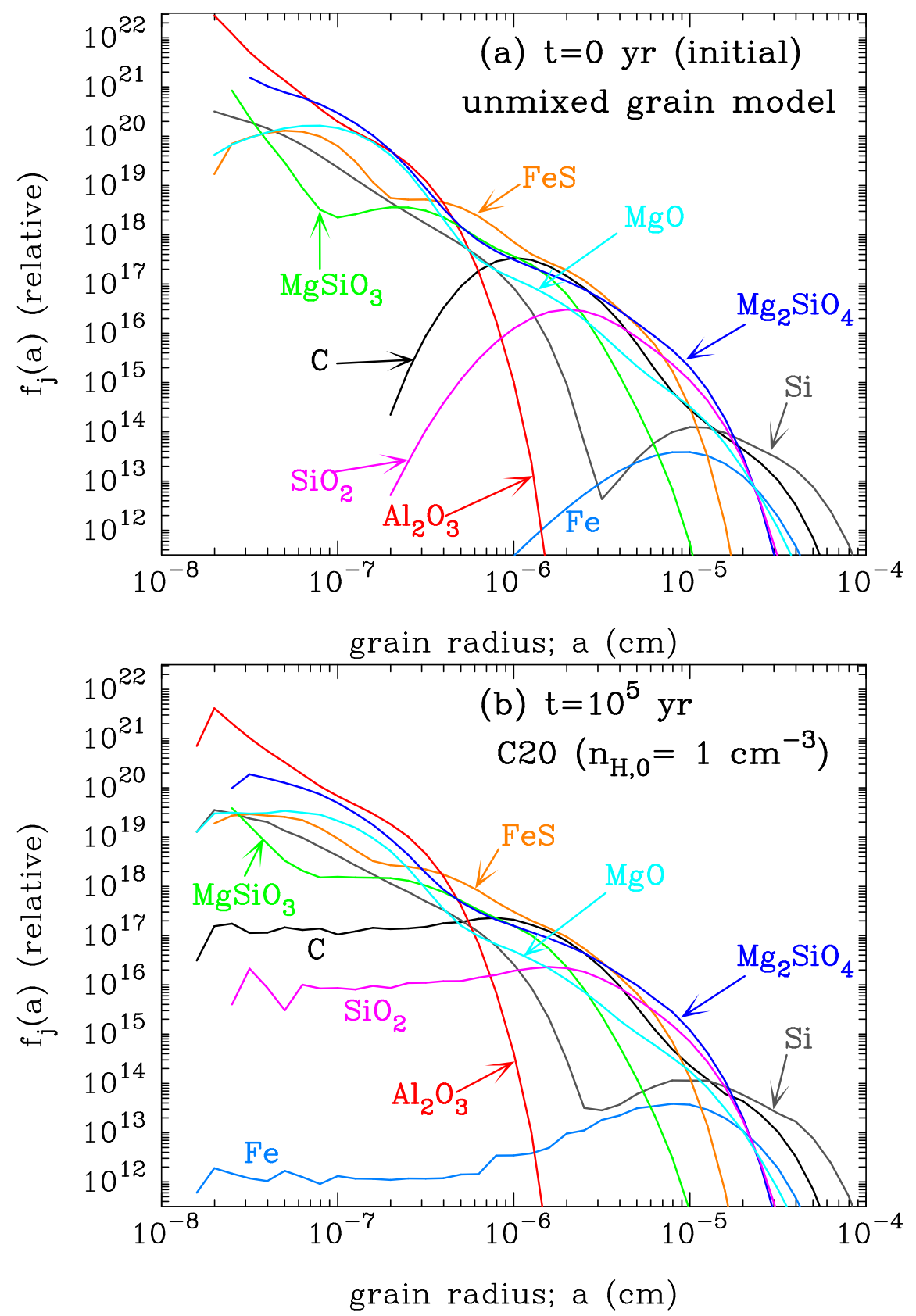

Fig. 6. - The size distribution of each dust species for the unmixed grain model; (a) for the initial size distribution before destruction and (b) for the size distribution obtained by the calculation of dust destruction for model C20 with $n_{\mathrm{H}, 0}=1 \mathrm{~cm}^{-3}$. See the electronic edition of the Journal for a color version of this figure. 

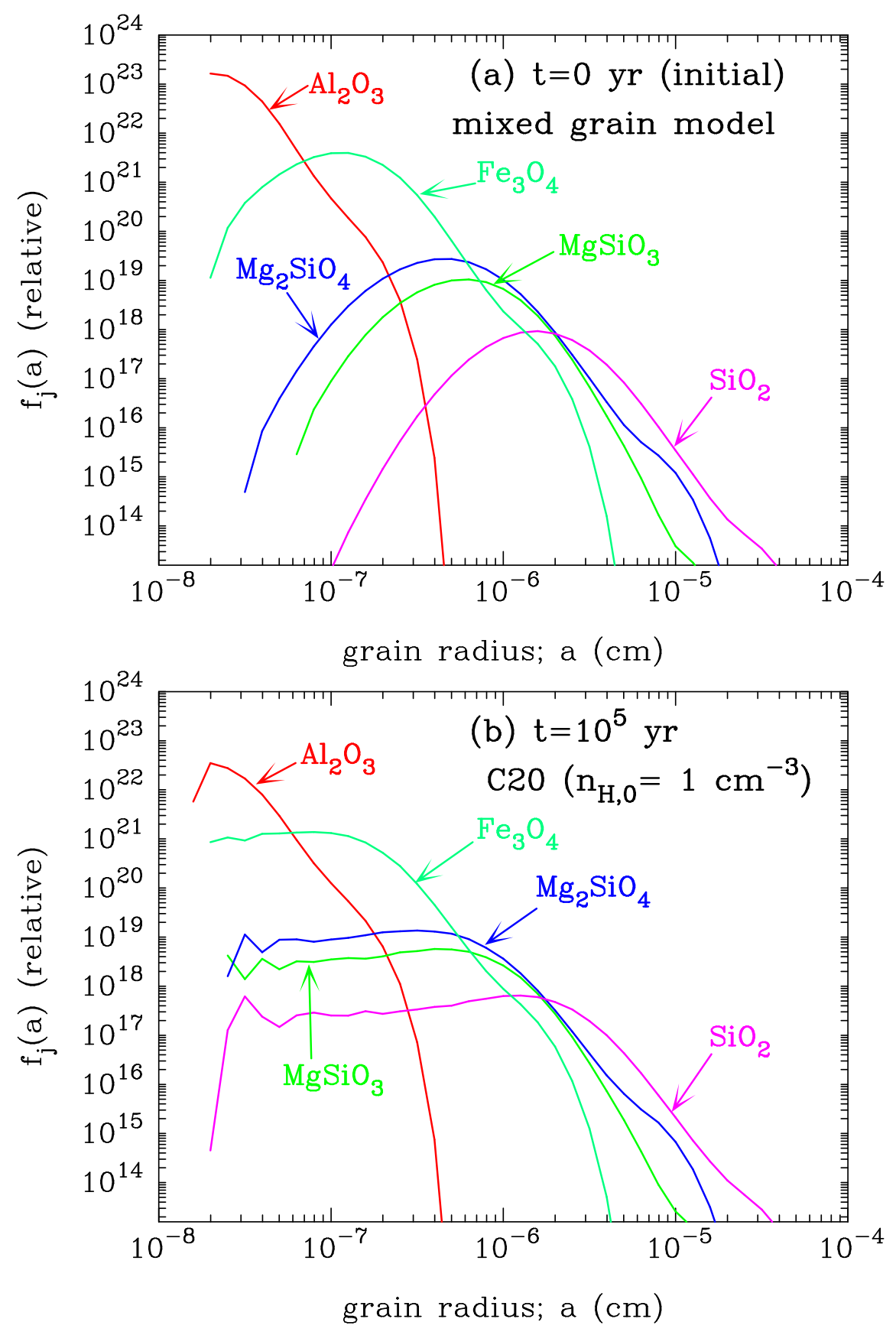

Fig. 7.- The size distribution of each dust species for the mixed grain model; (a) for the initial size distribution before destruction and (b) for the size distribution obtained by the calculation of dust destruction for model C20 with $n_{\mathrm{H}, 0}=1 \mathrm{~cm}^{-3}$. See the electronic edition of the Journal for a color version of this figure. 


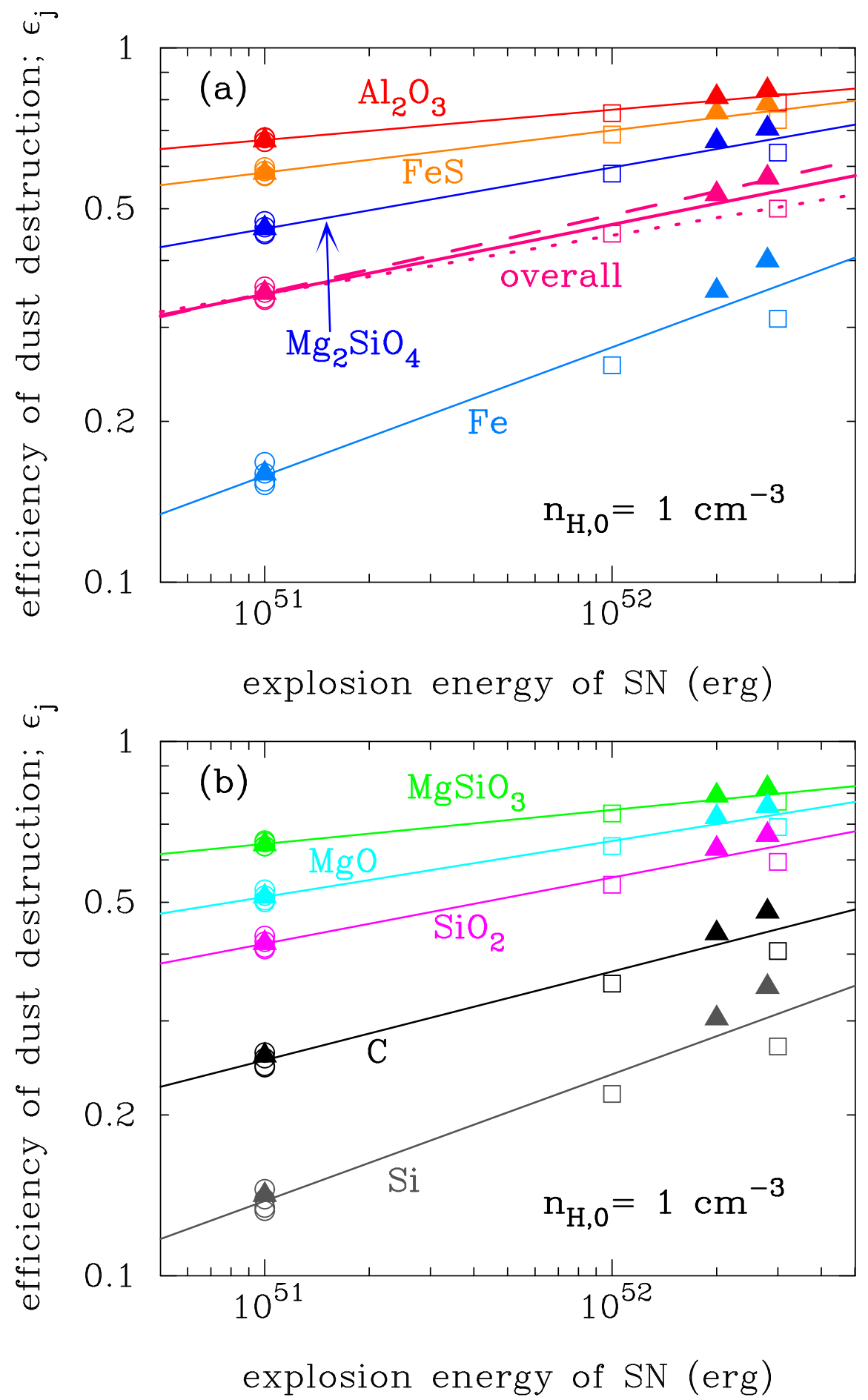

Fig. 8.- The efficiency of destruction of each grain species in the unmixed grain model calculated for $n_{\mathrm{H}, 0}=1 \mathrm{~cm}^{-3}$ as a function of SN explosion energy; (a) for $\mathrm{Al}_{2} \mathrm{O}_{3}, \mathrm{FeS}, \mathrm{Mg}_{2} \mathrm{SiO}_{4}$ and Fe grains and (b) for $\mathrm{MgSiO}_{3}, \mathrm{MgO}, \mathrm{SiO}_{2}, \mathrm{C}$ and $\mathrm{Si}$ grains. The $\mathrm{SN}$ models used for the calcularions are distinguished by open circles (CCSNe), open squares (HNe) and filled triangles (PISNe). Also, in (a), the overall efficiency of dust destruction is plotted. The linear solid lines for each grains species are the results calculated by the power-law formula given by Equation (A1) for all SNe. The dotted and dashed lines for the overall efficiencies of dust destruction are the results of calculations for SNe II and for PISNe, respectively. See the electronic edition of the Journal for a color version of this figure. 


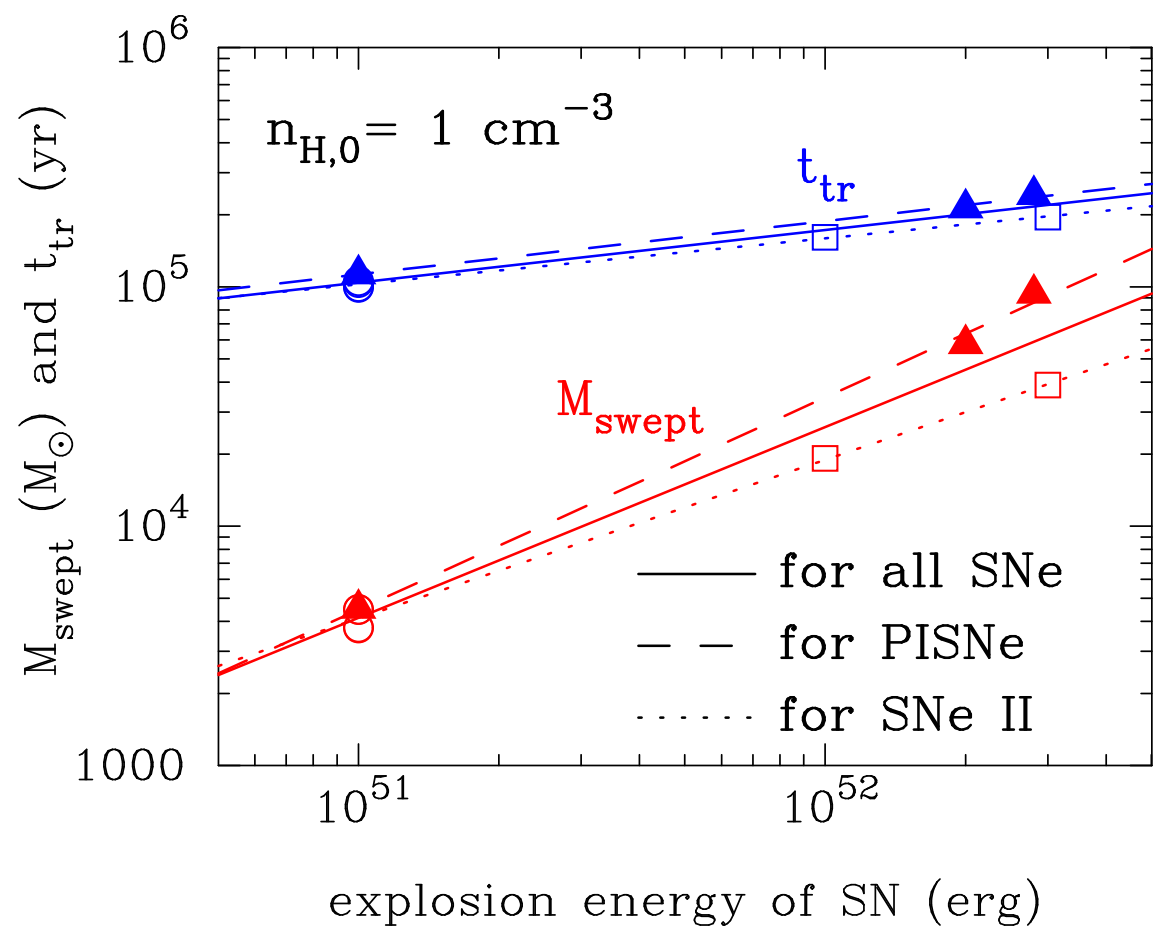

Fig. 9.- The mass of gas swept up by shock $M_{\text {swept }}$ and the truncation time $t_{\text {tr }}$ versus the explosion energy of $\mathrm{SN}$ for $n_{\mathrm{H}, 0}=1 \mathrm{~cm}^{-3}$. The results for CCSNe, HNe and PISNe are represented by open circles, open squares and filled triangles, respectively. The linear lines are the power-law formula approximated by Equation (A2) for all SNe (solid), SNe II (dotted) and PISNe (dashed). See the electronic edition of the Journal for a color version of this figure. 


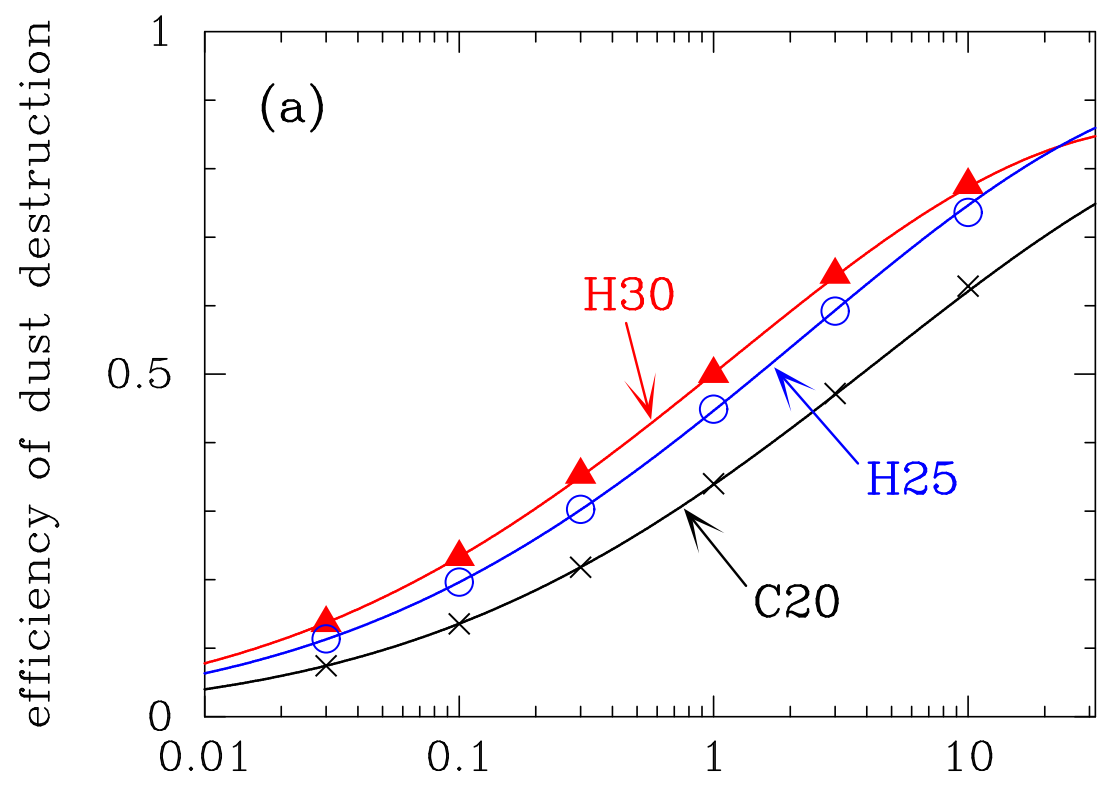

hydrogen number density in the ISM; $\mathrm{n}_{\mathrm{H}, 0}$

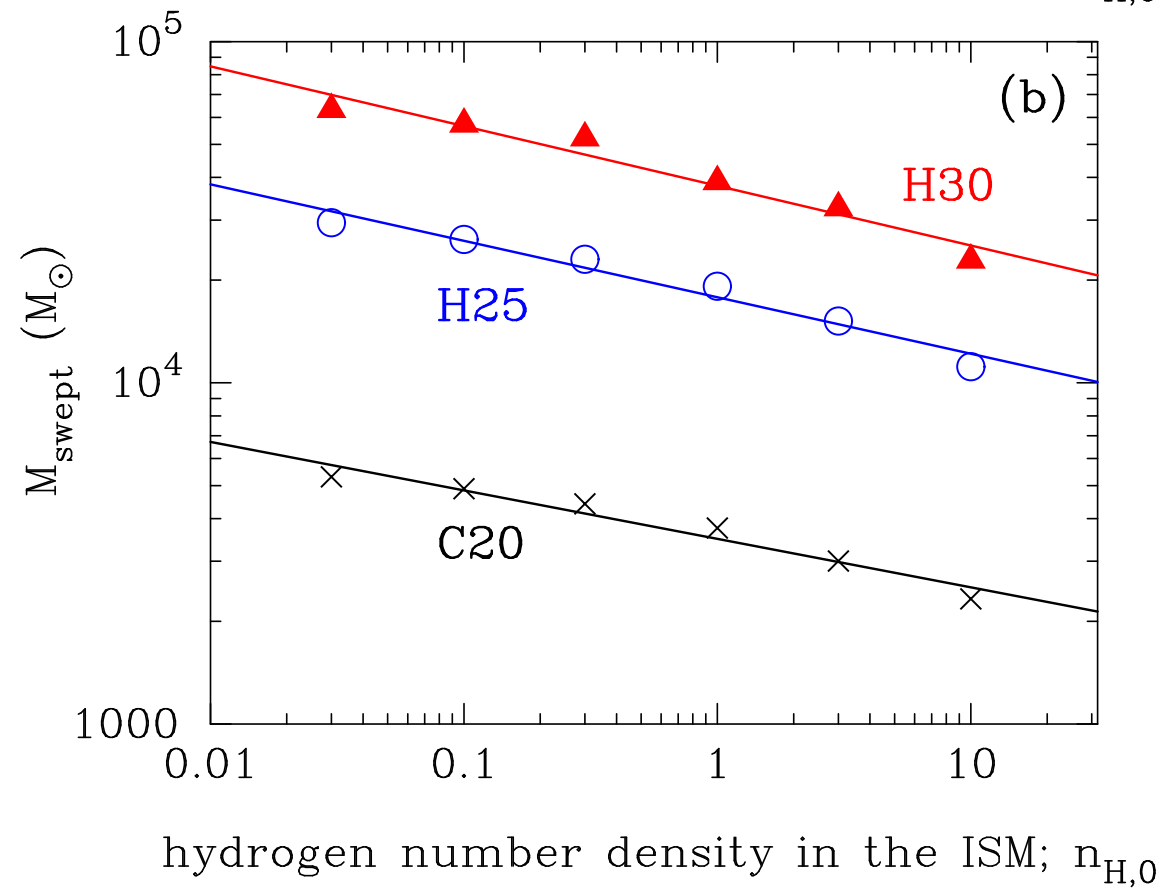

Fig. 10.- (a) The overall efficiency of dust destruction versus $n_{\mathrm{H}, 0}$ for C20 (crosses), H25 (open circles) and H30 models (filled triangles). The solid curves are calculated by the approximation formula by Equation (A3). (b) The mass of gas swept up by shock for C20 (crosses), H25 (open circles) and H30 models (filled triangles) as a function of $n_{\mathrm{H}, 0}$. The linear lines are the power-law approximation formula of $M_{\mathrm{swept}} \propto n_{\mathrm{H}, 0}^{g}$ with $g=-0.142 E_{51}^{0.063}$. See the electronic edition of the Journal for a color version of this figure. 


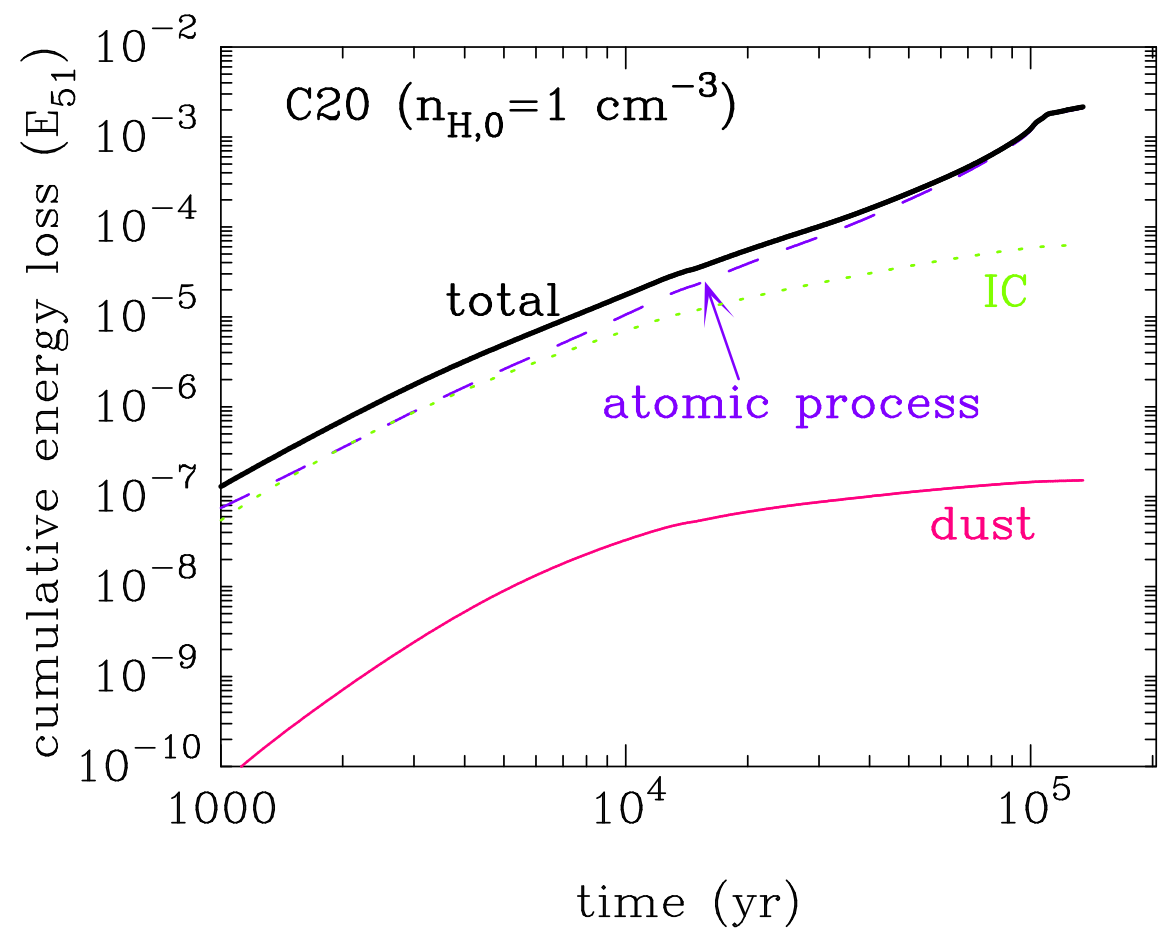

Fig. 11. - The time evolution of cumulative energy lost by the atomic process (dashed), the inverse Compton cooling (dotted) and the thermal emission from all dust grains (solid) in units of $E_{51}$ for the SN model C20, $n_{\mathrm{H}, 0}=1$ and $Z=10^{-4} Z_{\odot}$ at the redshift of $z=20$. The total energy loss is indicated by the thick solid curve. See the electronic edition of the Journal for a color version of this figure. 